\title{
Analysis and Optimization of a Protocol for Mobile Element Discovery in Sensor Networks
}

\author{
Francesco Restuccia, Giuseppe Anastasi, Marco Conti, and Sajal K. Das
}

\begin{abstract}
Recent studies have demonstrated that mobile elements (MEs) are an efficient solution to help decrease dramatically energy consumption in wireless sensor networks (WSNs). However, in most of cases, sensors use duty cycle schemes to save energy, and unless the ME mobility pattern is deterministic, each sensor node has to discover the presence of the ME in the nearby area before starting to exchange data with it. Therefore, in such wireless sensor networks with mobile elements (in short, WSN-MEs), the definition and analysis of a protocol for efficient ME discovery becomes of fundamental importance. In this paper, we propose an extensive performance analysis of an easy-to-implement, hierarchical discovery protocol for WSN-MEs, called Dual Beacon Discovery (2BD) protocol, taking into account stochastic, multi-path, variable speed ME mobility patterns. We also derive the optimal parameter values that minimize the energy consumption of sensor nodes, while guaranteeing the minimum node throughput required by the applications under consideration. Finally, we compare the 2BD protocol with a classical solution based on Periodic Listening (PL). Our results show that 2BD can exploit its hierarchical mechanism and thus significantly increase lifetime, especially when the ME discovery phase is relatively long.
\end{abstract}

Index Terms-Wireless Sensor Networks, Mobile Sink Discovery, Energy Efficiency, Throughput Optimization.

\section{INTRODUCTION}

W IRELESS Sensor Networks (WSNs) have emerged as an effective solution for a wide range of real-life applications, including monitoring (e.g., pollution prevention, precision agriculture, structural health of buildings), event detection (e.g., intrusions, fire/food emergencies), and target tracking (e.g., surveillance), just to name a few. In traditional application scenarios, sensor nodes are densely deployed over the sensing area, and data collection is carried out through multi-hop communication towards the sink node.

However, it is well-known that a multi-hop WSN may suffer from the so-called funnelling effect (or energy hole problem) [1]. In particular, the sensor nodes in the one-hop neighborhood of the sink are required to forward a significantly higher amount of data than those farthest apart. Thus, such nodes tend to spend more energy and hence exhaust their battery much earlier than other nodes. In applications in which replacing the sensors' batteries may be extremely difficult (and expensive), if not impossible (e.g., wireless underground/airborne sensor networks [2]), optimizing energy consumption (and thus network lifetime) becomes fundamental, and at the same time, extremely challenging.

- F. Restuccia and S.K. Das are with the Center for Research in Wireless Mobility and Networking (CReWMaN), Department of Computer Science and Engineering, University of Texas at Arlington, TX 76019 USA (e-mail: \{frestuc, das\}@uta.edu).

- Giuseppe Anastasi is with the Department of Information Engineering, University of Pisa, Italy (email: g.anastasi@iet.unipi.it).

- Marco Conti is with the Institute of Informatics and Telematics (IIT), National Research Council, Pisa, Italy (e-mail: marco.conti@iit.cnr.it).
Recently, the use of special mobile elements (MEs) has been suggested as an effective approach to mitigate the funneling or energy hole effect [1], [3], [4], and therefore increase network lifetime. MEs are special nodes that visit sensor nodes at regular intervals, gather sensed data, and transport them to the sink node. They are assumed to be powerful in terms of data storage and processing capabilities, and not energy constrained, due to the fact that their energy source can be replaced or recharged easily.

Specifically, MEs are exploited in many applications in which a dense deployment of sensor nodes may not be necessary (e.g., air quality monitoring in urban areas). In such applications, sensors can be strategically deployed in some specific locations and thus a sparse network configuration can be used to reduce deployment costs. In a sparse WSN, the distance between neighboring nodes is typically larger than the transmission range, so data collection can be carried out through MEs.

In general, unless the ME mobility pattern is well known, each sensor node has to discover the presence of the ME in the nearby area before starting to exchange data with it. Usually, a simple approach known as Periodic Listening (PL) is exploited for this purpose. In this approach, the ME emits periodic (very short) "beacon" messages to announce its presence in the area. At the same time, to save energy, sensor nodes wake up periodically for a very short time (i.e., using a duty cycle) to check for possible beacons from the ME [3]. Since a fixed duty cycle approach turns out to be energy-inefficient, adaptive solutions have been proposed [5], which dynamically adjust the duty cycle of sensor nodes, depending 
on the estimated probability that the ME is nearby. For the same reason, hierarchical discovery algorithms have also been proposed [7].

In order to allow a credible and massive deployment of wireless sensor networks using mobile elements (WSN-MEs), the design and the analysis of an easily implementable ME discovery protocol supposing complex ME mobility patterns becomes crucial. Additionally, it becomes necessary to investigate (through an extensive performance analysis) whether the protocol itself is able to guarantee quality of service (QoS) bounds, and at the same time, to derive the set of operating parameters leading to the desired trade-off between the ME discovery and energy efficiency.

The above discussions motivated us to thoroughly analyze the performance of an easy-to-implement, hierarchical discovery protocol for WSN-MEs, named the Dual Beacon Discovery (2BD) protocol [8]. The framework provides an analysis of the communication phase between the sensor node and the ME, and an algorithm for obtaining a given QoS bound on the system throughput, while minimizing the energy consumption at the sensor node. Our analytical results show that $2 \mathrm{BD}$ is able to provide a significant energy reduction with respect to a classical solution like PL, especially when the ME discovery phase becomes relatively long. Therefore, $2 \mathrm{BD}$ helps increase dramatically the lifetime of sensor networks using sink mobility, and by consequence, decreasing the maintenance costs of the sensor network itself.

The rest of the paper is organized as follows. Section 2 discusses the related works, while Section 3 briefly describes the 2BD protocol. Section 4 details the analytical framework used to analyze the performance of 2BD. The performance of 2BD and PL protocols are compared in Section 5. Finally, conclusions are drawn in Section 6.

\section{Related Work}

Since MEs have been used as an efficient solution for data collection in WSNs, many protocols and algorithms for ME discovery have been proposed in the literature. A comprehensive classification and detailed description of possible approaches to ME discovery is reported in [3]. Given the strict similarity to the problem investigated in this paper, related work on neighbor discovery algorithms for delay tolerant networks (DTNs) (also known as opportunistic networks (ONs)) will be surveyed as well.

As anticipated, the most commonly used approach for the ME discovery is Periodic Listening (PL), that exploits a fixed duty cycle to discover the presence of the $\mathrm{ME}$ in the nearby area. A fixed duty cycle scheme has been exploited in [9]. In particular, in this paper the authors proposed a probing mechanism in which the sensor node tries to discover the ME by emiting a beacon every time its radio is the ON state. In [10], the authors derive an analytical model of PL, and then propose an extensive performance analysis of the PL protocol itself. Despite the soundness of the work, the authors does not consider any optimization analysis regarding the PL protocol. Furthermore, the analytical model in [10] is oversimplified by the assumption that the $\mathrm{ME}$ can move on a linear trajectory and at a fixed speed only.

As regards DTNs and ONs, in the seminal work [11] Galluzzo et al. propose an analytical framework for studying the tradeoff between energy efficiency and time for the neighbor discovery process in ad-hoc networks. Similarly, in [12] the authors characterize the trade-off between the probability of a missed contact and the contact probing interval for stationary processes in DTNs. In [13], Izumikawa et al. propose an activation mechanism in which the activation interval is based on the surroundings, while in [14] Drula et al. investigate adaptive schemes for neighbor discovery in Bluetooth-enabled ad-hoc networks. In particular, their proposed schemes adaptively choose parameter settings depending on a mobility context to decrease the expected power consumption. Conversely from ours, none of these works consider the hierarchical approach as a solution to increase the lifetime of sensor nodes.

Specifically, differently from adaptive approaches [5], the efficiency of hierarchical schemes does not depend on the ME arrival / mobility pattern. Hierarchical discovery algorithms typically exploit two different radios, for wake-up / discovery and communication, respectively [6], [7]. Unlike the solutions in [6], [7], 2BD does not require multiple radio technologies - typically not available in current sensor platforms and can thus be implemented on any sensor platform.

The 2BD protocol was originally proposed in [8]. In our preliminary work [15], we analyzed the performance of 2BD considering linear mobility and fixed speed of the ME. This paper further extends our previous work by including in the framework the analysis of complex ME mobilities (i.e., multi-path, stochastic, variable-speed), and a completely new packet loss model, based on the distance between the sensor node and the ME. To the best of our knowledge, ours is the first work presenting a comprehensive and general analytical framework for the modeling of a hierarchical discovery protocol, able to capture complex ME mobility patterns and transmission areas, to analyze the joint ME discovery and data transfer process, as well as providing an optimization and performance analysis of the ME discovery protocol itself.

\section{DESCRIPTION OF 2BD PROTOCOL}

In this section, we briefly describe the 2BD protocol considered in our analysis. Hereafter, we will use the words 'sensor' and 'sensor node' interchangeably. 
In this protocol, sensors are assumed to switch between different duty cycles, according to a hierarchical approach. In particular, during the discovery phase, sensor nodes operate most of the time with a low duty cycle to save energy, and switch to a high duty cycle only when they perceive that the ME is about to enter their transmission range. Information about the actual ME location is provided to sensor nodes by the ME itself, through a periodic emission of two different beacon messages, namely Short-Range Beacons (SRBs) and Long-Range Beacons (LRBs). SRBs and LRBs are transmitted in an interleaved way, both with the same period $2 * T_{b i}$ such that $T_{b i}$ is the overall beacon period. However, they are transmitted with different transmission power, and thus convey different information.

Specifically, SRBs are transmitted with the same transmission-power level used during the communication phase for data exchange. Thus, they can be received within an area defined as communication area, and are aimed at notifying the sensor node the ME is within its transmission range, thus data exchange can actually take place. Conversely, LRBs are transmitted with a higher power than SRBs, and are used to inform the sensor node that the ME is approaching the communication area, and a contact could potentially occur shortly. Given the higher transmission power, LRBs can be received within a (much) larger area than the communication area, defined as discovery area. Hence the hierarchical nature of 2BD.

Please note that LRBs are received by the sensor node using the same radio interface used to receive SRBs and transmit data packets. Also note that, since the receive power of the radio is (usually) fixed to a given value [21], the dimension of the discovery area depends only on the transmission power of LRBs. Thereby, assuming that the ME is not an energyconstrained device, the transmission power of LRBs can be increased (or decreased) to enlarge (or shrink) the discovery area as necessary. The impact of the dimension of the discovery area on the performance of 2BD will be extensively analyzed in Section 5 .

Let us now detail the different phases of 2BD. As shown in Figure 1, during the discovery phase, the sensor node is initially in the LRB-Discovery state, and wakes up periodically (using a low duty cycle $\delta_{L}$ ) in order to check for possible beacons from the ME. Upon receiving a LRB, the sensor node transits to the SRB-Discovery state, increases its duty cycle to the high duty cycle value $\delta_{H}$, and waits for a SRB. To avoid wastage of energy, if a valid SRB is not received within a pre-defined timeout, the sensor node transits back to the LRB-Discovery (or Sleeping) state. Whenever a SRB is received (both in LRBDiscovery and SRB-Discovery state), the sensor node enters the Data Transfer state, increases the duty cycle to $100 \%$, and starts exchanging data with the ME. After transferring all its data, the sensor node transits to the LRB-Discovery state again in order to detect the next contact. However, if the sensor node has a (even partial) knowledge about the mobility pattern of the $\mathrm{ME}$, it can enter a Sleeping state in which the radio is put in sleep mode to save energy. In this case, the sensor node will enter the LRB-Discovery state some time before the estimated next arrival of the ME.

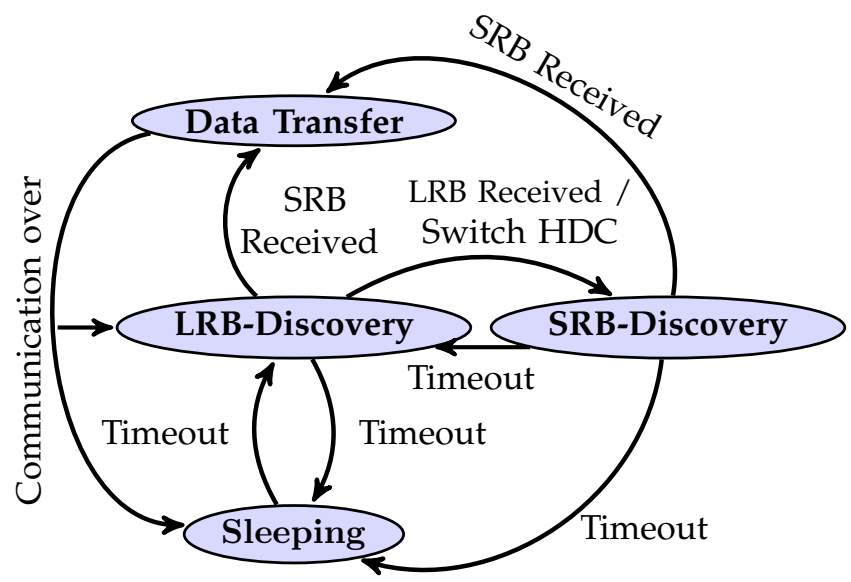

Figure 1: State diagram of a static sensor node using the 2BD protocol.

\section{Analytical framework of 2BD}

In this section, we present the analytical framework we developed to analyze $2 \mathrm{BD}$. This framework is quite generic, and can be extended to other discovery protocols for WSN-MEs. Firstly, let us describe the reference scenario shown in Figure 2, and the assumptions considered in our framework.

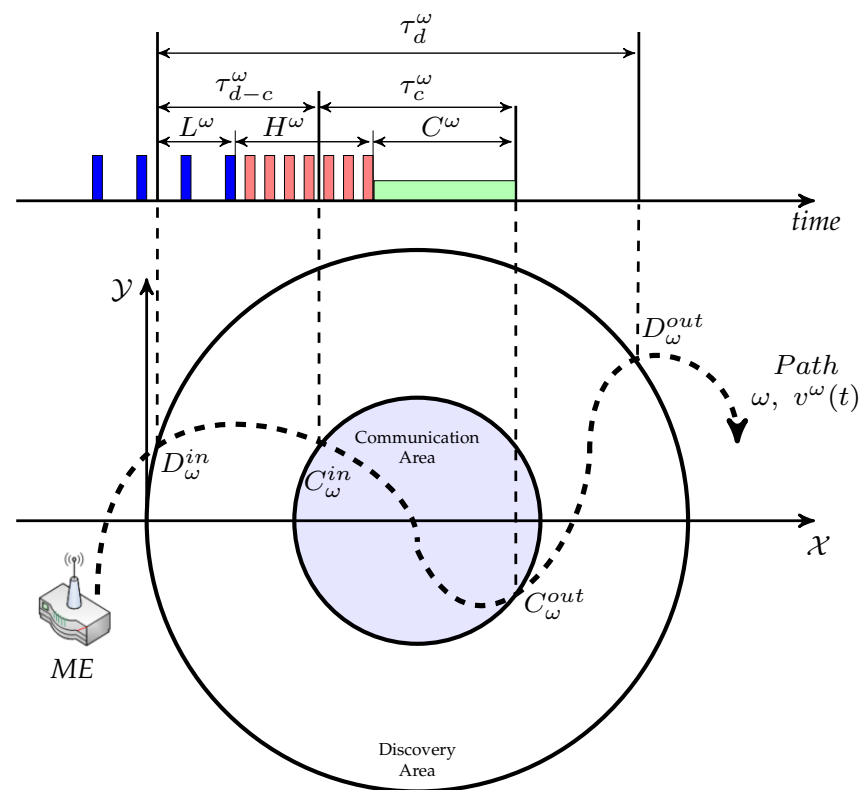

Figure 2: System model exemplified supposing a general curve $\omega$.

In Figure 2, the communication and discovery areas have been depicted as circles for the sake of graphical 
simplicity. However, our framework allows a description of the boundaries of the communication and discovery areas through generic curves so as to model the anisotropy of wireless transmission. Also, irregular boundaries can model scenarios in which some sub-areas may not be available for data exchange (e.g., presence of obstacles between the ME and the sensor node along the path).

In our analysis, we consider a sparse WSN in which the distance between neighboring nodes is very large (much larger than the discovery range), and, without losing in generality, we also assume that there is a single ME in the sensing area. Therefore, at any time, the ME can communicate with at most one sensor node. Also, we will assume that the sensor node is deployed along the ME path in a way that both LRBs and SRBs can be received (i.e., false activations cannot occur). Finally, we will assume that the ME can move through the sensing area along several paths, each defined by a curve $\omega$ present in the set $\Omega$ of possible ME paths. For a path along curve $\omega$, we suppose that the ME can move at a variable speed $v^{\omega}(t)$ at time $t$. Note that these assumptions do not lead to loss of generality of the model, as the information about the ME mobility pattern can be plugged into the model in function of the specific deployment scenario of $2 \mathrm{BD}$. In particular, the sets $\Omega$ and $v^{\omega}(t)$ should represent, respectively, all possible paths the ME could choose to pass through (e.g., a set of different streets or railways in an urban environment), and the relative speeds (e.g., for different streets or railways the ME could experience different traffic conditions, and thus, could change speed accordingly). Also, we will suppose that the ME mobility is not controllable, i.e., the ME cannot stop and wait until the sensor node wakes up and all data has been transferred (e.g., the ME is a vehicle like a tram or a bus).

Let the maximum time interval the $\mathrm{ME}$ can stay in the communication area and discovery area be denoted by $\tau_{c}^{\omega}$ and $\tau_{d}^{\omega}$, respectively. Additionally, let the maximum time interval the ME can be in the discovery area before entering the communication area be denoted by $\tau_{d-c}^{\omega}$. Note that all these quantities depend on the curve $\omega$ and the ME speed $v^{\omega}(t)$. Due to space limitations, the derivation of the quantities $\tau_{c}^{\omega}, \tau_{d}^{\omega}$ and $\tau_{d-c}^{\omega}$ based on the curve $\omega$ and the related ME speed $v^{\omega}(t)$ are presented in the Appendix.

As depicted in Figure 2, the interaction between the sensor node and the ME can be divided into four phases. In detail, the first phase ranges from when the sensor node enters the LRB-Discovery state (thus starting the discovery process) to when the ME enters the discovery area. The next phase, of duration $L^{\omega}$, is terminated by the reception of the first LRB by the sensor node. This increases the duty cycle, as shown in Figure 2. The third phase corresponds to the time interval $H^{\omega}$ between the reception of the first LRB and the reception of a valid SRB. Finally, the communication phase starts with the reception of a valid SRB, and ends when all data have been transmitted or the ME has exited the communication area. Hence, the overall time spent by the sensor node in the LRB-Discovery and SRB-Discovery states, calculated from when the ME enters the discovery area to the actual ME discovery, is given by $L^{\omega}+H^{\omega}$. Let the time available for data communication be denoted as $C^{\omega}$. The goal of our analysis is to derive the distributions $l(i)$ and $h(i)$ of $L$ and $H$, respectively (by considering all the curves $\omega$ in $\Omega$ ), as well as the average energy $\bar{E}_{d s c}$ and $\bar{E}_{c o m}$ spent by the sensor node in the discovery and communication phases, respectively.

As far as packet loss is concerned, we consider a generic packet loss model in which the probability $p^{\omega}\left(t_{k}\right)$ to lose a SRB or message at time $t_{k}$ is a function of the distance between the ME and the sensor node, i.e., defining $x_{M E}^{\omega, t_{k}}$ as the position of the $\mathrm{ME}$ on a given curve $\omega$ at a given time $t_{k}$, and $x_{S N}$ as the (fixed) position of the sensor node,

$$
p^{\omega}\left(t_{k}\right)=\mathcal{F}\left\{d\left(x_{M E}^{\omega, t_{k}}, x_{S N}\right)\right\}
$$

Now, given the specific curve $\omega \in \Omega$, and the average ME speed $\bar{v}_{t_{k}}^{\omega}$ from $t=0$ to $t_{k}$, the position $x_{M E}^{\omega, t_{k}}$ of the ME at a given time $t_{k}$ can be approximated by

$$
x_{M E}^{\omega, t_{k}}: \int_{D^{i n}}^{x_{M E}^{\omega, t_{k}}} \sqrt{1+\omega^{\prime}(x)^{2}} d x=t_{k} \cdot \bar{v}_{t_{k}}^{\omega}
$$

Therefore, given the position $x_{M E}^{\omega, t_{k}}$, a function $\mathcal{F}$, and a specific distance function $d(\cdot)$, the calculation of $p_{t_{k}}^{\omega}$ follows directly from Equation (1). Clearly, the packet loss model will depend on the specific scenario in which the ME discovery protocol will be analyzed. In the following, we assume that LRBs are always accepted (since they just signal the presence of the ME in the region), while corrupted messages/SRBs received within the communication area are discarded.

\subsection{BD Discovery Phase Analysis}

This subsection derives a stochastic model of the 2BD discovery phase, based on a Discrete Time Markov Chain (DTMC). The purpose of this analysis is to calculate the distribution of both the LRB-Discovery time $(L)$ and SRB-Discovery time $(H)$, as well as the residual time available for communication after discovery.

The analysis is split into two main phases. First, the state of the radio of the sensor node (i.e., $\mathrm{ON}$ or OFF) over time is derived, by keeping into consideration its duty cycle. Second, the beacon reception process is modeled by characterizing the state transitions of the sensor node, based on the probability that a beacon sent by the ME will be correctly received by the sensor node. Note that, in the following, we first derive the LRB and SRB distributions by considering a single curve $\omega \in \Omega$. The derivation of the global distribution is calculated next considering all curves in $\Omega$. From 
now on, every quantity has to be referred to the curve $\omega$, unless otherwise stated.

Table 1: Main symbols used in the discovery phase analysis.

\begin{tabular}{|c|l|}
\hline Symbol & Description \\
\hline \hline$\Omega$ & Set of curves describing the ME mobility \\
$\omega$ & Curve in $\Omega$ describing a particular ME path \\
$\delta_{L}$ & Low duty cycle (LDC) \\
$\delta_{H}$ & High duty cycle (HDC) \\
$T_{b i}$ & Beacon Interval \\
$T_{b d}$ & Beacon Duration \\
$T_{O N}$ & Active Time \\
$T_{O F F}^{L D C}$ & LDC inactivity time \\
$T_{O F F}^{H D C}$ & HDC inactivity time \\
$T_{L D C}$ & LDC total time \\
$T_{H D C}$ & HDC total time \\
$\tau R S(t)$ & Current Radio State at time t \\
$H$ & SRB-Discovery r.p. \\
$L$ & LRB-Discovery r.p. \\
$t_{0}^{L}$ & Time of first LRB transmission into Discovery Area \\
$t_{0}^{S}$ & Time of first SRB transmission into Discovery Area \\
$t_{L}$ & Average LRB-discovery time \\
$t_{H}$ & Average SRB-discovery time \\
$X(k)$ & State probability vector \\
$\mathbb{P}\{C D\}$ & Complete Discovery probability \\
$\mathbb{P}\{P D\}$ & Partial Discovery probability \\
$\mathbb{P}\{C M\}$ & Complete Miss probability \\
$\mathbb{P}\{P M\}$ & Partial Miss probability \\
$P_{R X}$ & Receiving radio power \\
$P_{T X}$ & Transmission radio power \\
$P_{S L}$ & Sleep radio power \\
&
\end{tabular}

Given beacon transmissions do not depend on when the ME enters into the discovery area (assumed as $t=0$ ), the initial LRB transmission within the discovery area is generally affected by a random offset, with respect to the time origin. Let $t_{0}^{L}$ be the time instant at which the ME transmits the first LRB while in the discovery area. $t_{0}^{L}$ is a random variable (r.v.) uniformly distributed in $\left[0,2 T_{b i}\right)$. By the definition of $t_{0}^{L}$ and the beacon periodicity, it follows that the actual instants $t_{i}^{L}$ of subsequent LRB transmissions are expressed as $t_{i}^{L}=t_{0}^{L}+i \cdot 2 T_{b i}$, with $i \in\left[1, N_{L}-1\right]$, where $N_{L}$ is the maximum number of LRBs the ME can send while in the discovery area. By definition, it follows that

$$
N_{L}= \begin{cases}\left\lceil\frac{\tau_{d}}{2 \cdot T_{b i}}\right\rceil & \text { if } \tau_{d}-\left(\left\lceil\frac{\tau_{d}}{2 \cdot T_{b i}}\right\rceil-1\right) \cdot 2 \cdot T_{b i}>t_{0}^{L} \\ \left\lfloor\frac{\tau_{d}}{2 \cdot T_{b i}}\right\rfloor & \text { otherwise }\end{cases}
$$

Additionally, since the transmissions of SRBs and LRBs are interleaved and separated by a period $T_{b i}$, the time $t_{0}^{S}$ of the first SRB transmission in the discovery area, the maximum number $N_{S}$ of SRBs the ME can send while in the discovery area, and the instants of subsequent SRB transmissions $t_{i}^{S}$ can be derived as follows.

$$
\begin{gathered}
t_{0}^{S}= \begin{cases}t_{0}^{L}-T_{b i} & \text { if } t_{0}^{L} \geq T_{b i} \\
t_{0}^{L}+T_{b i} & \text { if } t_{0}^{L}<T_{b i} .\end{cases} \\
N_{S}= \begin{cases}\left\lceil\frac{\tau_{d}}{2 \cdot T_{b i}}\right\rceil & \text { if } \tau_{d}-\left(\left\lceil\frac{\tau_{d}}{2 \cdot T_{b i}}\right\rceil-1\right) \cdot 2 \cdot T_{b i}>t_{0}^{S} \\
\left\lfloor\frac{\tau_{d}}{2 \cdot T_{b i}}\right\rfloor & \text { otherwise } \\
t_{i}^{S}=t_{0}^{S}+i \cdot 2 T_{b i}, i \in\left[1, N_{S}-1\right]\end{cases}
\end{gathered}
$$

Let $t_{k}$ denote the time instant when the $k$-th beacon is received, for $k \in\left[1, N_{B}\right]$, where $N_{B}=N_{S}+N_{L}$ is the total number of transmitted beacons. To characterize the radio state at beacon reception times $t_{k}$, we introduce the function $R S(t)$ that assumes the value $\mathrm{ON} / \mathrm{OFF}$ if the radio is active/inactive at time $t$. We denote by $R S_{0}$ the radio state (i.e., ON/OFF) at time $t=0$. Also, we indicate by $r_{0}$ the residual time in which the radio will remain in state $R S_{0}$, starting from time $t=0$. For simplicity, we define $T_{L D C} \triangleq T_{O N}+T_{O F F}^{L D C}$ and $T_{H D C} \triangleq T_{O N}+T_{O F F}^{H D C}$ as the period of low and high duty cycle, respectively. Finally, we define an auxiliary function $R_{L R B}(t)$ such as $R_{L R B}(t)=$ True if a LRB has already been received at time $t$. The following Claim holds.

Claim 1. The state of the sensor node at time $t_{k}$ is given by

$$
\begin{gathered}
\begin{array}{c}
R S\left(t_{k}\right) \\
R S_{0}=O N \\
R_{L R B}\left(t_{k}\right)=\text { False }
\end{array}= \begin{cases}O N & 0 \leq t_{k}^{\prime}<r_{0} \\
O F F & r_{0} \leq t_{k}^{\prime}<r_{0}+T_{O F F}^{L D C} \\
O N & r_{0}+T_{O F F}^{L D C} \leq t_{k}^{\prime}<\end{cases} \\
\begin{array}{c}
R S\left(t_{k}\right) \\
R S_{0}=O F F \\
R_{L R B}\left(t_{k}\right)=\text { False }
\end{array}= \begin{cases}O F F & 0 \leq t_{k}^{\prime}<r_{0} \\
O N & r_{0} \leq t_{k}^{\prime}<r_{0}+T_{O N} \\
O F F & r_{0}+T_{O N} \leq t_{k}^{\prime}<\end{cases} \\
R S\left(t_{k}\right) \\
T_{L D C}\left(t_{k}\right)=\text { True } \\
\text { where } t_{k}^{\prime}= \begin{cases}O N & 0 \leq t_{k}^{\prime \prime}<T_{O N} \bmod T_{L D C} \\
\text { OFF } & T_{O N} \leq t_{k}^{\prime \prime}<T_{H D C} \\
\text { and } t_{k}^{\prime \prime}=\left(t_{k}-t_{R-L R B}\right) \bmod T_{H D C}\end{cases}
\end{gathered}
$$

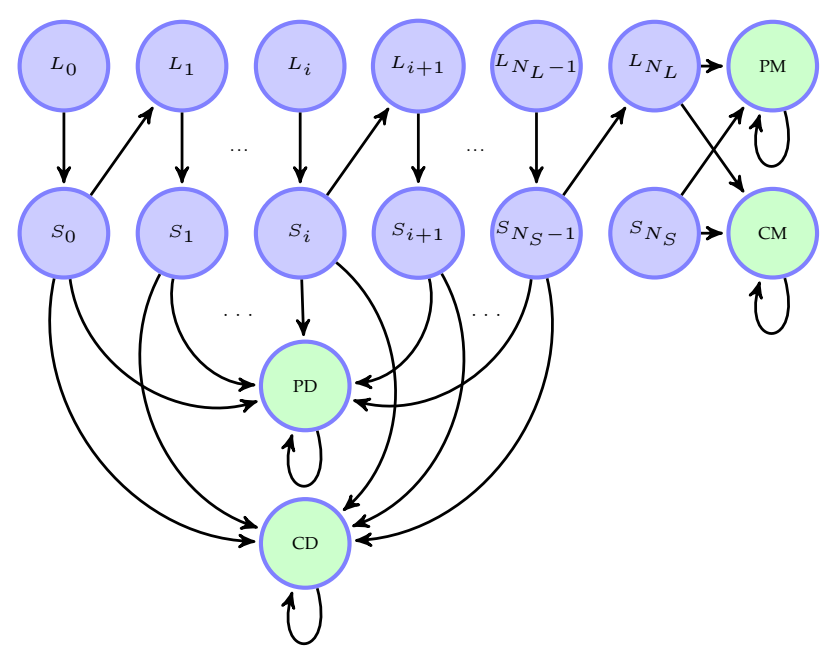

Figure 3: DTMC State diagram when $t_{0}^{S}>t_{0}^{L}$.

Due to space limitations, the proof of Claim 1 is shown in the Appendix. Once the radio state at beacon reception times has been fully characterized, we can now model the beacon reception process. The evolution of the system at beacon reception times can be modeled by a Discrete Time Markov Chain (DTMC), where each macro state $L i$ (resp. $S_{i}$ ) corresponds to 


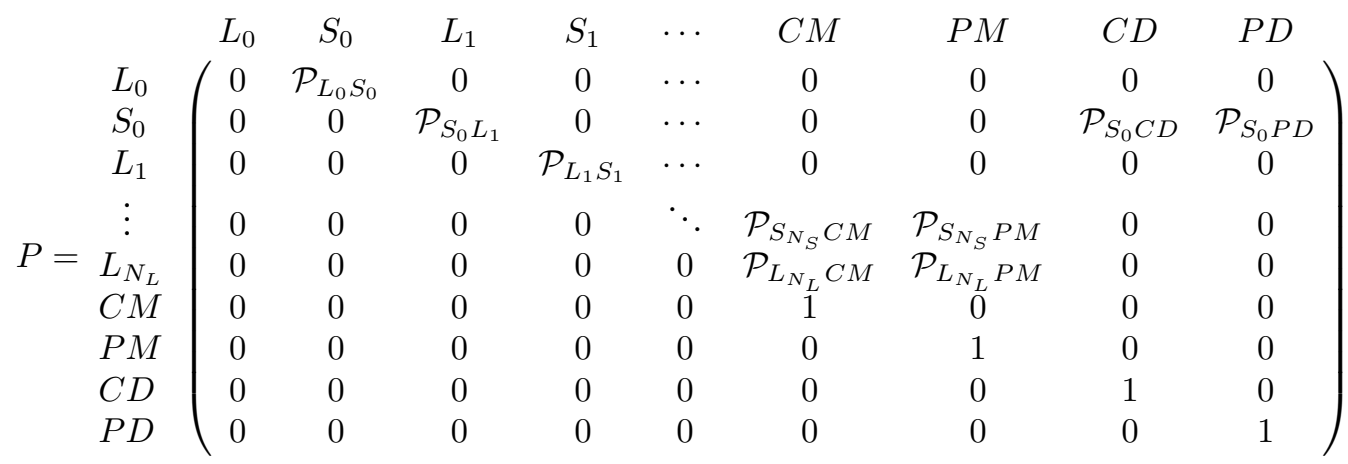

several different states, all representing the same LRB (resp. SRB) reception process. Specifically, we denote by $L_{i}$ (resp. $S_{i}$ ) the macro state that keeps track of all possible radio states just before the reception of the $i$-th LRB (resp. SRB). Details on the composition of those macro states will be provided below. Figure 3 shows the DTMC when the first beacon emitted into the discovery area is a LRB (i.e., $t_{0}^{S}>t_{0}^{L}$ ), while Equation (3) shows the transition matrix $P$ corresponding to Figure 3. Due to space limitations, the DTMC corresponding to the case $t_{0}^{S}<t_{0}^{L}$ is shown in the Appendix, along with a brief description thereof. The transition matrix $Q$ corresponding to such DTMC is very similar to $P$, and can be derived straightforwardly from its state diagram by following the structure of $P$.

Table 2: Absorbing states of the DTMCs.

\begin{tabular}{|c|l|l|l|}
\hline State & Description & LRB Received & SRB Received \\
\hline \hline$C D$ & Complete Discovery & YES & YES \\
$P D$ & Partial Discovery & NO & YES \\
$C M$ & Complete Miss & NO & NO \\
$P M$ & Partial Miss & YES & NO \\
\hline
\end{tabular}

Let us discuss first the DTMC depicted in Figure 3 . Initially, the system is in state $L_{0}$, and eventually converges to one of the absorbing states as defined in Table 2. The Complete Discovery (CD) and Partial Discovery $(P D)$ characterize a success, in sense that the ME is discovered by the sensor node. The Complete Miss (CM) and Partial Miss (PM), on the other hand, characterize a failure, in which the sensor node does not discover the ME. The different absorbing states are characterized by different energy consumptions. From a generic state $L_{i}$, the system always transits to state $S_{i}$, irrespective of the $i$-th LRB reception status, to keep the timing of the DTMC constant. Instead, from state $S_{i}$, the system can evolve into the following states:

- $L_{i+1}$, if the $i$-th SRB has been missed because the radio is OFF, i.e., $R S\left(t_{i}^{S}\right)=O F F$, or a transmission error has occurred, with probability $p\left(t_{i}^{S}\right)$;

- CD, if an LRB has already been received, i.e., $R_{L R B}\left(t_{i}^{L}\right)=$ 'True';

- PD, otherwise.
Finally, at the end of the process, i.e., when in state $L_{N_{L}}$ or $S_{N_{S}}$, the system transits to the absorbing state PM or CM, depending on whether an LRB has been already received or not.

Let us now focus on the transition probability matrix $P$ and let us detail the expression of blocks $\mathcal{P}_{X Y}$. Given time is discretized with slots $\Delta$, the residual time in a radio state can assume $M$ different values, where $M=\left\lceil\left(T_{O N}+T_{L D C}^{O F F}\right) / \Delta\right\rceil$. As anticipated, since the initial radio state and the initial residual time can assume all possible values, sub-blocks $P_{X Y}$ in matrix $P$ have size $M \times M$ and keep track of all possible transition probabilities from the generic state $X$ to the generic state $Y$, assuming all possible radio states at time $t_{k}$ of the evaluation of state $X$. Let $s x_{k}, k=1,2, \ldots, M$ be the set of all possible radio states at time $t_{k}$, obtained by using the $R S(t)$ function calculated at time $t_{k}$ and by considering all the $M$ initial radio states. It follows the elements of the $\mathcal{P}_{X Y}$ blocks are defined as

$$
b_{X Y}^{\left(s x_{k}\right)}=\mathbb{P}\left\{Y \mid X\left(s x_{k}\right)\right\}, k=1,2, \ldots, M
$$

Below we detail the expression of all $p_{X Y}$ sub-blocks.

$$
\begin{aligned}
& b_{S_{i} L_{j}}^{\left(s x_{k}\right)}=\left\{\begin{array}{ll}
p^{\omega}\left(t_{k}\right) & j=i+1 \wedge s x_{k}=O N \\
1 & j=i+1 \wedge s x_{k}=O F F \\
0 & j \neq i+1 \\
t_{0}^{S}>t_{0}^{L} & \\
p^{\omega}\left(t_{k}\right) & j=i, s x_{k}=O N \\
1 & j=i, s x_{k}=O F F \\
0 & j \neq i \\
t_{0}^{S}<t_{0}^{L} &
\end{array}\right\}
\end{aligned}
$$

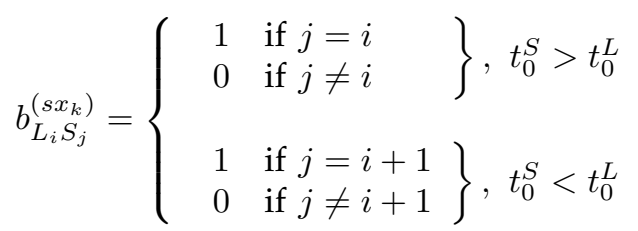

$$
\begin{aligned}
& b_{S_{i} P D}^{\left(s x_{k}\right)}= \begin{cases}1-p^{\omega}\left(t_{k}\right) & R_{L R B}\left(t_{k}\right)={ }^{\prime} \text { False' }^{\prime}, s x_{k}=O N \\
0 & \text { otherwise }\end{cases} \\
& b_{S_{i} C D}^{\left(s x_{k}\right)}= \begin{cases}1-p^{\omega}\left(t_{k}\right) & R_{L R B}\left(t_{k}\right)=\text { 'True' }^{\prime}, s x_{k}=O N \\
0 & \text { otherwise }\end{cases}
\end{aligned}
$$




$$
\begin{aligned}
& b_{L_{N_{L}} C M}^{\left(s x_{k}\right)}= \begin{cases}1 & R_{L R B}\left(t_{k}\right)={ }^{\prime} \text { False }^{\prime} \\
0 & \text { otherwise }\end{cases} \\
& b_{L_{N_{L}} P M}^{\left(s x_{k}\right)}= \begin{cases}1 & R_{L R B}\left(t_{k}\right)=\text { 'True' }^{\prime} \\
0 & \text { otherwise }\end{cases} \\
& b_{S_{N_{S}} C M}^{\left(s x_{k}\right)}= \begin{cases}1 & R_{L R B}\left(t_{k}\right)={ }^{\prime} \text { False' }^{\prime} \\
0 & \text { otherwise }\end{cases} \\
& b_{S_{N_{S}} P M}^{\left(s x_{k}\right)}= \begin{cases}1 & R_{L R B}\left(t_{k}\right)={ }^{\prime} \text { True' } \\
0 & \text { otherwise }\end{cases}
\end{aligned}
$$

where $i \in\left[0, N_{S}-1\right], j \in\left[0, N_{L}-1\right], k \in[1, M]$. Let $N_{T}=N_{S}+N_{L}+2$ be the number of states of the DTMC, except for the absorbing states. Let $\mathbf{X}^{(0)}$ be the initial state probability vector of the sensor node and $\mathbf{X}^{(k)}$ be the state probability vector after the $k$-th beacon transmission, with $k \in\left[1, N_{T}\right]$. Thus,

$$
\begin{aligned}
& \mathbf{X}^{(k)}=\left(\begin{array}{lllll}
X_{0}^{(k)} & X_{1}^{(k)} & \cdots & X_{N_{T}-1}^{(k)} & X_{N_{T}}^{(k)}
\end{array}\right) \\
& \mathbf{X}^{(0)}=\left(\begin{array}{lllll}
X_{0}^{(0)} & 0 & \cdots & 0 & 0
\end{array}\right)
\end{aligned}
$$

Note that only the $X_{0}^{(0)}$ component of the initial state vector is not zero. This is because, when the ME enters into the contact area, the sensor node is waiting for the first beacon. Following the theory of DTMCs, the state vector $\mathbf{X}^{(k+1)}$ can be derived from $\mathbf{X}^{(k)}$ as

$$
\underset{k \in\left[0, N_{T}\right]}{\mathbf{X}^{(k+1)}}= \begin{cases}\mathbf{X}^{(k)} \cdot P & \text { if } t_{0}^{S}>t_{0}^{L} \\ \mathbf{X}^{(k)} \cdot Q & \text { if } t_{0}^{S}<t_{0}^{L}\end{cases}
$$

Now we can derive the time spent by the sensor node in low and high duty cycles. To this end, we define two random variables, namely $L$ and $H$, denoting the time spent by the sensor node (starting from the time origin) until the reception of the first LRB and SRB, respectively. We denote by $l^{\omega}(i)$ and $h^{\omega}(i)$, respectively, the probability mass functions (p.m.f.) of $L$ and $H$ referred to a curve $\omega \in \Omega$. The distribution $h^{\omega}(i)$, given that the first SRB transmission occurs at time $t_{0}^{S}$, denoted by $h^{\omega}\left(i \mid t_{0}^{S}\right)$, is

$$
\begin{gathered}
h^{\omega}\left(i \mid t_{0}^{S}\right) \\
\mathbb{P}\left\{\begin{array}{l}
\left.S=t_{i}^{S} \mid t_{0}^{S}\right\} \\
t_{0}^{S}>t_{0}^{L}
\end{array}=\left\{\begin{array}{l}
X_{N_{T}}^{(2)}+X_{N_{T}-1}^{(2)}, i=0 \\
\left(X_{N_{T}}^{(2 i+2)}+X_{N_{T}-1}^{(2 i+2)}\right)- \\
\left(X_{N_{T}}^{(2 i)}+X_{N_{T}-1}^{(2 i)}\right), \\
i \in\left[1, N_{S}-1\right] \\
h^{\omega}\left(i \mid t_{0}^{S}\right) \\
t_{0}^{S}<t_{0}^{L}
\end{array}\right\}\left\{\begin{array}{l}
X_{N_{T}}^{(1)}+X_{N_{T}-1}^{(1)}, i=0 \\
\left(X_{N_{T}}^{(2 i+1)}+X_{N_{T}-1}^{(2 i+1)}\right)- \\
\left(X_{N_{T}}^{(2 i-1)}+X_{N_{T}-1}^{(2 i-1)}\right), \\
i \in\left[1, N_{S}-1\right] \\
0, \text { otherwise }
\end{array}\right.\right.
\end{gathered}
$$

Note that $X_{N_{T}-1}^{(k)}$ and $X_{N_{T}}^{(k)}$ represent the cumulative probability that the ME has been detected after $k$ beacon transmissions, by either a complete discovery or a partial discovery. In Equation (6) (resp. Equation (7)), $h^{\omega}\left(i \mid t_{0}^{S}\right)$ includes the probability of having either a complete discovery, corresponding to $X_{N_{T}-1}(k)$, or a partial discovery, corresponding to $X_{N_{T}}(k)$. Note that in Equation (6) (resp. Equation (7)), only even (resp. odd) iterations are considered, since SRBs are interleaved with LRBs. Before eliminating the dependency from $t_{0}^{S}$ in Equations (6) and (7), we now derive $l^{\omega}\left(i \mid t_{0}^{S}\right)$. The following Claim holds.

Claim 2. The distribution $l^{\omega}(i)$, given that the first SRB transmission occurs at time $t_{0}^{S}$, denoted by $l^{\omega}\left(i \mid t_{0}^{S}\right)$, is

$$
l^{\omega}\left(i \mid t_{0}^{S}\right)=\mathbb{P}\left\{L=t_{i}^{L} \mid t_{0}^{S}\right\}= \begin{cases}1 & \text { if } i=i^{*} \\ 0 & \text { otherwise }\end{cases}
$$

PROOF. Since we assumed that LRBs are never discarded, given the initial radio state $\left(S_{0}, r_{0}\right)$ and the first SRB transmission time $t_{0}^{S}$, the time of the first LRB reception can be deterministically derived as the $t_{i *}^{L}$ such that the radio is ON, i.e., $R S\left(t_{i *}^{L}\right)=$ True. Thus, $l^{\omega}\left(i \mid t_{0}^{S}\right)$ is a Kronecker delta function centered to the index $i^{*}$ of the first LRB received by the sensor node.

Finally, in order to eliminate the dependency of $h^{\omega}(i)$ and $l^{\omega}(i)$ from $t_{0}^{S}$, we need to consider all possible values of $t_{0}^{S}$ and the corresponding probabilities. Since we are assuming that time is discrete, with time slot $\Delta$, let us denote by $\Lambda \equiv\{0, \Delta, \cdots, n \cdot \Delta\}, n=\left\lfloor T_{b i} / \Delta\right\rfloor$ the set of possible values that can be assumed by $t_{0}^{S}$ (corresponding to each random offset). Since each value in $\Lambda$ can occur with probability $\Delta / T_{b i}$, it derives by the law of total probability that the p.m.f. of the LRB discovery time $l^{\omega}(i)$ and the SRB discovery time $h^{\omega}(i)$ can be expressed as

$$
\begin{gathered}
h^{\omega}(i)=\frac{\Delta}{T_{b i}} \cdot \frac{\sum_{t_{0}^{S R B} \in \Lambda} h^{\omega}\left(i \mid t_{0}^{S R B}\right)}{\sum_{i=1}^{N_{S}}\left(\sum_{t_{0}^{S R B} \in \Lambda} h^{\omega}\left(i \mid t_{0}^{S R B}\right)\right)} \\
l^{\omega}(i)=\frac{\Delta}{T_{b i}} \cdot \frac{\sum_{t_{0}^{S R B} \in \Lambda} l^{\omega}\left(i \mid t_{0}^{S R B}\right)}{\sum_{i=1}^{N_{L}}\left(\sum_{t_{0}^{S R B} \in \Lambda} l^{\omega}\left(i \mid t_{0}^{S R B}\right)\right)}
\end{gathered}
$$

Until now, we have analyzed a single curve $\omega$. In order to derive the global distribution $h(i)$ and $l(i)$, we need to consider all possible $\omega \in \Omega$, and the related probability that the ME will choose the path $\omega$, denoted by $\mathbb{P}\{w\}$. Following the law of total probability, the distributions $h(i)$ and $l(i)$ can be derived as

$$
\begin{aligned}
& h(i)=\sum_{\omega \in \Omega} \mathbb{P}\{w\} \cdot h^{\omega}(i) \\
& l(i)=\sum_{\omega \in \Omega} \mathbb{P}\{w\} \cdot l^{\omega}(i)
\end{aligned}
$$




\subsection{Data Transfer Analysis}

Now we focus on the communication phase and derive (i) the average number of bytes per detected contact correctly transferred to the ME, and (ii) the average total energy consumed by the sensor during the data transfer phase. By doing so, we will use the p.m.f.s $d(i)$ and $l(i)$ derived beforehand.

For the sake of generality, we assume (as in [17], [18]) that the data exchange between the sensor node and the ME is carried out through a simple ARQ (Automatic Repeat reQuest) protocol with selective retransmissions that is briefly described below.

Upon receiving a valid beacon, the sensor node enters the communication state and starts transmitting messages of fixed duration $T_{m}$ to the ME. On the other hand, the ME replies with ACK messages "piggybacked" in periodic beacons. Specifically, the sensor nodes transmits a window of $N_{m}$ messages back to back and, then waits for the periodic ACK. Here $N_{m}$ is set such that the $N_{m}$-th message is received by the ME just before the transmission of the periodic ACK/beacon). Note that ACKs specify which messages in the previous window have been received correctly by the ME. Then, the sensor node transmits another window of $N_{m}$ messages including both messages not acknowledged by the previous ACK and new messages. If the ACK is missed, the sensor nodes retransmits all message sent in the previous window. Finally, as the sensor node cannot know when the ME leaves the communication area, it implicitly assumes that the communication with the ME is lost when it misses $N_{a c k}$ number of consecutive ACKs.

Next, let us derive the amount of messages correctly sent by the sensor node to the ME during a data transfer phase. Then, we will use this information to derive the throughput of the system. Since we consider a lossy channel for SRBs and data messages, every message being transmitted into the communication area will experience its own loss probability.

On the other hand, since the message duration is short, it is reasonable to assume that the message loss introduced by the channel will be constant during a message transmission, i.e., the $i$-th message in the window starting at time $t$ will experience a constant loss probability $p^{\omega}\left(t+i \cdot T_{m}\right)$. We assume that the queue at the sensor node has infinite size and is always full, i.e., the application has always data to send. We also assume that data and acknowledgement messages have a fixed duration of $T_{m}$ and $T_{b d}$, respectively, and $B_{m}$ the bytes transmitted with each message. Given $T_{m}, T_{b i}$ and $T_{b d}$, the number of messages in a window can be derived as $N_{m}=\left\lfloor\frac{T_{b i}-T_{b d}}{T_{m}}\right\rfloor$.

In order to get the total number of messages correctly transmitted, we need to derive the number of windows that can be transmitted into a data transfer phase. Clearly, this depends on the residual contact time available for data transmission, i.e., $C$. Using the equations derived in the previous section, it follows that the number of potential windows using the residual contact time available, i.e., $N_{w}$ is given by $N_{w}=\left\lfloor\frac{C}{T_{b i}}\right\rfloor$.

Now we will derive the number of messages correctly transferred by the sensor node to the $\mathrm{ME}$ in a single ARQ window. Let define as $t=0$ the time in which the data transfer phase starts, i.e., the time in which a SRB is correctly received by the sensor node. Thus, the starting time $t_{j}$ of the $j$-th window is

$$
t_{j}=(j-1) \cdot T_{b i}, \quad j=1,2, \cdots, N_{w}
$$

We focus on a single window starting at the generic time $t_{j}$. We define $R_{i j}$ as the r.v. denoting the actual transmission status of the $i$-th message transmitted from the static node in the $j$-th window, i.e., 1 if successful and 0 if failed. Clearly, we can model $R_{i j}$ as a Bernoulli r.v., $i \in\left[1, N_{m}\right]$. In particular, the distribution of $R_{i j}$, is given by

$$
r_{i j}(m)=\left\{\begin{array}{lc}
1-p^{\omega}\left(t_{j}+(i-1) \cdot T_{m}\right) & m=1 \\
p^{\omega}\left(t_{j}+(i-1) \cdot T_{m}\right) & m=0 \\
0 & \text { otherwise }
\end{array}\right.
$$

Thus, the r.v. $R_{j}$ denoting the total number of messages received by the ME in the $j$-th window can be derived as

$$
R_{j}=\sum_{i=1}^{N_{m}} R_{i j}, \quad j=1,2, \cdots, N_{w}
$$

The p.m.f of $R_{j}$ can be obtained as the convolution of the $N_{m}$ p.m.f.s $r_{i j}(m)$ :

$$
r_{j}(m)=\otimes_{i=1}^{N_{m}} r_{i j}(m), \quad j=1,2, \cdots, N_{w}
$$

Furthermore, we denote by $A_{j}$ the r.v. representing the number of messages correctly transferred to the ME in the $j$-th window when an ARQ-based scheme is used. Thus, $A_{j}$ represents the number of messages acknowledged by the ME in the $j$-th window. As said before, we consider a selective retransmission scheme, i.e., acknowledgements notify the sensor node which messages sent in the last window have been correctly received by the ME. Therefore, the reception of the acknowledgement has to be accounted as well. In particular, the messages within a window are correctly transferred if they are successfully received by the ME and the corresponding acknowledgement is not lost. As for $R_{i j}$, we can model $A_{j}$ as a Bernoulli r.v.. In particular, the distribution of $A_{j}$, i.e., $a_{j}(m)$ is obtained as

$$
a_{j}(m)=\left\{\begin{array}{lc}
1-p^{\omega}\left(t_{j}+T_{b i}-T_{b d}\right) & m=1 \\
p^{\omega}\left(t_{j}+T_{b i}-T_{b d}\right) & m=0 \\
0 & \text { otherwise }
\end{array}\right.
$$

Since the messages sent in the current window need to be acknowledged in order to be correctly received, it follows that $A_{j}=R_{j} \cdot A_{j}$. Furthermore, since $R_{j}$ and $A_{j}$ are independent r.v., it follows that the average number of messages transmitted in a ARQ-based scheme, using the generic $j$-th window starting at time $t_{j}$ and supposing a queue always full, is derived 
as $N_{j}=\mathbb{E}\left\{A_{j}\right\}=\mathbb{E}\left\{R_{j}\right\} \cdot \mathbb{E}\left\{A_{j}\right\}$. Then, we can derive the total number $\left(N_{t o t}\right)$ of correctly received messages by the ME from the sensor node as $N_{t o t}=\sum_{j=1}^{N_{w}} N_{j}$.

Thus, the average energy spent during communication phase can be derived as

$$
\begin{aligned}
\bar{E}_{c o m}= & \left\{P_{T X} \cdot\left(N_{w}+N_{a c k}\right) \cdot N_{m} \cdot T_{m}+\right. \\
& \left.P_{R X} \cdot\left(N_{w}+N_{a c k}\right) \cdot T_{b d}\right\} \cdot \mathbb{P}\{D\}
\end{aligned}
$$

In detail, sensor node sends $\left(N_{w}+N_{a c k}\right)$ windows of $N_{m}$ messages of duration $T_{m}$. Then, waits for $\left(N_{w}+N_{a c k}\right)$ acknowledge messages, of duration $T_{b d}$. Clearly, both the throughput and the energy spent during communication have to be accounted when the communication actually occurs. Hence, both quantities have to be multiplied by the overall ME discovery probability, i.e., $\mathbb{P}\{D\}$. Finally, we can derive the system throughput $\theta$ as

$$
\theta=N_{\text {tot }} \cdot B_{m} \cdot \mathbb{P}\{D\}
$$

\subsection{Analysis of Discovery-Phase Energy Con- sumption}

In the following, we will derive the energy consumed by the sensor node in the discovery phase. Since the discovery process can have four different outcomes, we derive the Average Energy Consumption for: (i) Complete Discovery $\left(\bar{E}_{C D}\right)$, (ii) Partial Discovery $\left(\bar{E}_{P D}\right)$, (iii) Complete Miss $\left(\bar{E}_{C M}\right)$, and (iv) Partial Miss $\left(\bar{E}_{P M}\right)$. By definition of $L$ and $H$ and using Equations (9) and (10), the average time spent by the sensor node in low duty cycle $\left(t_{L}\right)$ and high duty cycle $\left(t_{H}\right)$ can respectively be obtained as $t_{L}=\mathbb{E}[L], t_{H}=\mathbb{E}[H-L]$. The equations for the aforementioned energies are defined in the following equations.

$$
\begin{gathered}
\bar{E}_{C D}=\begin{array}{l}
P_{S L} \cdot\left\{\left(1-\delta_{L}\right) \cdot t_{L}+\left(1-\delta_{H}\right) \cdot t_{H}\right\}+ \\
P_{R X} \cdot\left\{\delta_{L} \cdot t_{L}+\delta_{H} \cdot t_{H}\right\}
\end{array} \\
\bar{E}_{P D}=P_{S L} \cdot\left(1-\delta_{L}\right) \cdot t_{H}+P_{R X} \cdot \delta_{L} \\
\bar{E}_{C M}=P_{S L} \cdot\left(1-\delta_{L}\right) \cdot T_{O U T}+P_{R X} \cdot \delta_{L} \cdot T_{O U T} \\
\bar{E}_{P M}=P_{S L} \cdot\left\{\left(1-\delta_{L}\right) \cdot t_{L}+\left(1-\delta_{H}\right) \cdot T_{O U T}\right\}+ \\
P_{R X} \cdot\left\{\delta_{L} \cdot t_{L}+\delta_{H} \cdot T_{O U T}\right\} .
\end{gathered}
$$

Where $P_{S L}$ (resp. $P_{R X}$ ) denotes the power consumed by the sensor node in sleep (resp. receive) mode, $\delta_{L}$ (resp. $\delta_{H}$ ) is the low (resp. high) duty cycle, and $T_{O U T}$ is the maximum time the sensor node remains active after receiving a LRB. In case of a Complete Discovery $\left(\bar{E}_{C D}\right)$, the sensor node remains in low duty cycle for a total time $t_{L}$, and then switches to high duty cycle for a time $t_{H}$. On the other hand, when a Partial Discovery $\left(\bar{E}_{P D}\right)$ occurs, the sensor node remains in low duty cycle for a total time $t_{H}$, and then begins to communicate with the ME. In case of a Complete Miss $\left(\bar{E}_{C M}\right)$, the sensor node remains in low duty cycle for a time equal to $T_{O U T}$. Finally, in case of a Partial Miss $\left(\bar{E}_{P M}\right)$, the sensor remains in low duty cycle for a total time $t_{L}$ and then switches to high duty cycle until the timeout expires. Therefore, the average total energy spent in the discovery phase is derived as

$$
\begin{aligned}
\bar{E}_{d s c}= & \bar{E}_{C D} \cdot \mathbb{P}\{C D\}+\bar{E}_{P D} \cdot \mathbb{P}\{P D\}+ \\
& \bar{E}_{C M} \cdot \mathbb{P}\{C M\}+\bar{E}_{P M} \cdot \mathbb{P}\{P M\}
\end{aligned}
$$

\subsection{Optimization Analysis}

To perform an integrated analysis of both the discovery and communication processes, we consider the performance metric Energy Per Byte Acknowledged (EPBA), which is the average total energy spent by the sensor node per acknowledged byte and given by

$$
\bar{E}_{b}=\frac{\bar{E}_{d s c}+\bar{E}_{c o m}}{N_{t o t} \cdot B_{m}}
$$

Now we derive the optimal parameter values $\delta_{L}^{o p t}$ and $\delta_{H}^{o p t}$ that minimize the EPBA, while guaranteeing the minimum throughput $\theta_{\min }$ required by the application. For simplicity, we define $\theta\{\delta\}$ and $\bar{E}_{b}(\delta)$, respectively, as the system throughput obtained from Equation (13) and the EPBA obtained by considering the set of duty cycle parameters $\delta=\left\{\delta_{L}, \delta_{H}\right\}$. From these definitions, it follows the optimization problem can be formulated as

$$
\underset{\delta}{\min }\left\{\bar{E}_{b}(\delta): \theta\{\delta\} \geq \theta_{\min }\right\}
$$

To solve the optimization problem through Equation (16), we used a modified version of the gradient descent algorithm [19]. For the sake of space, the algorithm has been presented in the Appendix.

\section{Performance Analysis}

In this section, we compare the performance of the 2BD protocol with that of a traditional, fixed duty cycle discovery protocol, namely Periodic Listening (PL). To this end, we derived an analytical model for PL similar to the one described above for 2BD, and performed the same optimization analysis described in Section 4.7, thus obtaining the optimal duty cycle. First, let us show the assumptions upon which our analysis will be based. Then, we present the results obtained from the optimizations, considering different network parameters. In the following, the performance comparison will be carried out in terms of the energy saving $E S$ provided by the 2BD protocol with respect to the PL protocol. Therefore,

$$
E S=\frac{\bar{E}_{b}^{P L}-\bar{E}_{b}^{2 B D}}{\bar{E}_{b}^{P L}}
$$

In order to evaluate the impact of $2 \mathrm{BD}$ and $\mathrm{PL}$ on the residual time available for data exchange, we also derive the metric residual contact ratio $(C R)$, defined as the ratio between the time available for data exchange $C$ and the total nominal time available for data exchange $C_{\max }$.

Unless stated otherwise, we will use the parameter values shown in Table 3. Starting from this basic configuration, we will vary some of the parameters to 
Table 3: Parameters chosen for analysis.

\begin{tabular}{|l|l|}
\hline Parameter & Value \\
\hline \hline Receive power $\left(P_{R X}\right)$ & $35.46 \mathrm{~mW}$ \\
Transmission power $\left(P_{T X}\right)$ & $31.32 \mathrm{~mW}$ \\
Sleep mode power $\left(P_{S L}\right)$ & $0.36 \mu W$ \\
Beacon interval $\left(T_{b i}\right)$ & $100 \mathrm{~ms}$ \\
Beacon duration $\left(T_{b d}\right)$ & $1 \mathrm{~ms}$ \\
Time slot $(\Delta)$ & $10 \mathrm{~ms}$ \\
Communication range $(r)$ & $70 \mathrm{~m}$ \\
Discovery range $(R)$ & $200 \mathrm{~m}$ \\
Bitrate & $250 \mathrm{Kbit} / \mathrm{s}$ \\
Message size $\left(B_{m}\right)$ & $20 \mathrm{bytes}$ \\
Message duration $\left(T_{m}\right)$ & $0.64 \mathrm{~ms}$ \\
$\alpha, \beta, \gamma, \lambda$ & $35,5,20,1 / 15$ \\
\hline
\end{tabular}

investigate their individual impact on $E S$. The values communication parameters in Table 3 are inspired from the IEEE 802.15.4 standard [20], while the radio parameters are assumed to be the same of a Chipcon CC2420 radio transceiver [21]. Since the sensor must be able to receive at least one beacon when it wakes up, the value $T_{O N}$ has been set equal to the minimum value $T_{b i}+T_{b d}$ in all experiments. Without loss of generality, we will assume that both communication and discovery areas are modeled as circumferences having radii $r$ and $R$ (defined as communication and discovery range, respectively). Since an outdoor environment is the most credible deployment for WSNMEs, as far as the packet loss model is concerned, we adopted real packet error rate (P.E.R.) measurements in an outdoor environment derived in [16]. In detail, we fixed the packet size length at 20 bytes and the communication range at $r=70 \mathrm{~m}$. The SRB (beacon in PL) transmission range has been set to $70 \mathrm{~m}$ as well in both protocols.

Regarding the ME mobility scenario, we decided to investigate patterns in which the ME is bounded to choose from $N$ different paths $\omega_{i}$, each one chosen with a uniform probability $\left(\mathbb{P}\left\{w_{i}\right\}=\frac{1}{N}\right)$. For example, this mobility pattern can be easily mapped into scenarios in which the ME collects data from the sensor node by moving through a main road composed by $N$ different lanes. In order to exemplify these mobility scenarios, and to characterize real applications of WSN-MEs, we fixed $N=4$ and defined a linear and a curvilinear scenario. In detail, in the linear scenario, the ME moves along paths characterized by straight lines, and described by the equation

$$
\omega_{i}=\alpha+i \cdot \beta, i=0,1,2,3
$$

Conversely, in the curvilinear scenario, the ME moves along paths characterized by cosinusoidal curves:

$$
\omega_{i}=\gamma \cdot \cos (x \cdot \lambda)+\alpha+i \cdot \beta, i=0,1,2,3
$$

where $x$ is the $\mathrm{x}$-axis coordinate in the C.C.S. $(\mathcal{X}, \mathcal{Y})$ described in Figure 2. In particular, the linear and curvilinear mobility patterns can schematize well scenarios in which the WSN-MEs is deployed on a urban road and a mountain road, respectively. The values of $\alpha, \beta, \gamma, \lambda$ are specified in Table 3. Hereafter, we will refer to the term 'discovery state', respectively, as the LRB-discovery phase of 2BD and the beacon discovery phase of PL.

Due to its hierarchical nature, 2BD is more suitable to random mobility scenarios where the arrival time of the ME at the sensor node is completely unknown. However, it may also be appealing also for scenarios where the ME mobility pattern is predictable. Typically, in such scenarios the arrival time of the ME can be predicted with some uncertainty and, hence, the sensor node must be in the discovery state for some time, while waiting for the ME. Obviously, in a random mobility scenario no information about the ME mobility pattern is available and, hence, the sensor node has to be always in the discovery state (i.e., the waiting time tends to infinity). In order to assess the energy performance of both 2BD and PL protocols, we assume that the sensor node has some information about inter-contact times that can be predicted based on the past history. However, the sensor node does not know the exact arrival time of the $\mathrm{ME}$, thus, it enters the discovery state some time in advance with respect to the predicted arrival time. The time interval from when the sensor node goes to the discovery state to when the ME enters into the communication area will be referred to as the waiting time in the rest of the paper. Clearly, the waiting time is related to the uncertainty in the arrival time prediction (the higher the uncertainty, the larger the waiting time). In a random mobility scenario, where no information is available, the sensor node has to be always in the discovery state and, hence, the waiting time tends to infinity. In our analysis, under the same operating conditions, the waiting time is the same for both PL and $2 \mathrm{BD}$, since the communication range is unchanged.

Before presenting the experimental results, it is worthwhile describing the differences between the curvilinear and the linear ME mobility scenario. In particular, using the parameters shown in Table 3, the curvilinear scenario presents (i) a higher average ME permanence time in the discovery area before entering the communication area (i.e., $15.48 \mathrm{~s}$ vs. $12.23 \mathrm{~s}$ ), and (ii) a higher average maximum nominal contact time (i.e., 11.86 s vs. $10.91 \mathrm{~s})$.

\subsection{Results}

Figure 4 and Figure 5 show the energy savings provided by $2 \mathrm{BD}$, with respect to $\mathrm{PL}$, as a function of the waiting time and for different values of (minimum) throughput, expressed in kilobytes per contact, supposing a linear and a curvilinear ME mobility. Also, Table 4 to 7 report the optimal duty cycles obtained optimizing 2BD and PL in the linear and curvilinear scenario, as well as the LRB-Discovery and SRB-Discovery time (i.e., $L$ and $H$ ) and the related residual contact ratio. In all tables, the upper (reps. lower) side corresponds to the results regarding linear (resp. curvilinear) mobility. 


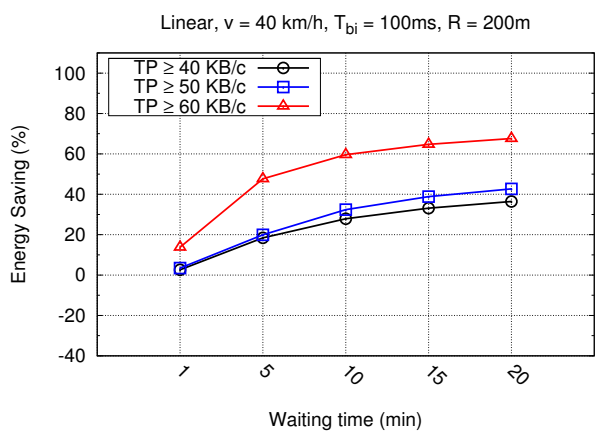

Figure 4: Energy savings of 2BD in function of throughput, Linear ME mobility.

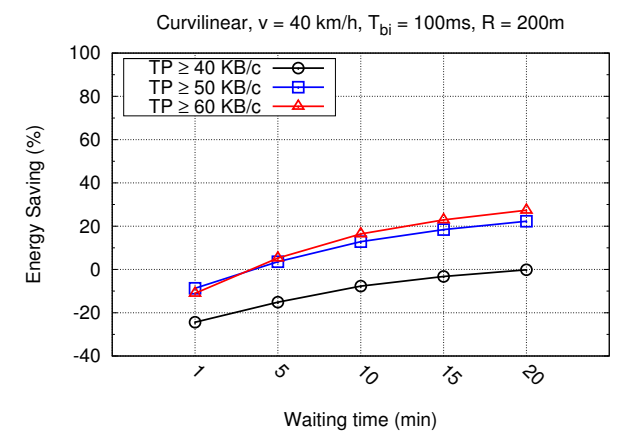

Figure 5: Energy savings of 2BD in function of throughput, Curvilinear ME mobility.

Table 4: Optimal duty cycle Values in function of $\theta$ (Kbytes per contact), for linear (topside) and curvilinear (bottomside) ME mobility.

\begin{tabular}{|c|c|c|c|c|c|c|l|}
\hline$\theta$ & $\delta^{o p t}$ & $\delta_{L}^{o p t}$ & $\delta_{H}^{o p t}$ & $L$ & $H$ & $C R_{2 b d}$ & $C R_{p l}$ \\
\hline$\geq 40$ & 1.5 & 0.8 & 6.4 & 12.54 & 15.78 & $65.50 \%$ & $66.48 \%$ \\
$\geq 50$ & 2.4 & 1.0 & 7.8 & 10.04 & 14.93 & $77.57 \%$ & $77.86 \%$ \\
$\geq 60$ & 6.5 & 1.4 & 9.2 & 5.01 & 13.68 & $89.31 \%$ & $90.53 \%$ \\
\hline$\theta$ & $\delta^{o p t}$ & $\delta_{L}^{o p t}$ & $\delta_{H}^{o p t}$ & $L$ & $H$ & $C R_{2 b d}$ & $C R_{p l}$ \\
\hline$\geq 40$ & 0.8 & 0.6 & 5.5 & 16.72 & 22.15 & $59.75 \%$ & $59.21 \%$ \\
$\geq 50$ & 1.0 & 0.6 & 5.7 & 16.72 & 20.42 & $61.65 \%$ & $60.22 \%$ \\
$\geq 60$ & 1.3 & 0.7 & 6.8 & 14.33 & 19.58 & $68.33 \%$ & $69.44 \%$ \\
\hline
\end{tabular}

Conversely from what expected, Figure 5 emphasizes that 2BD achieves a lower energy saving, with respect to $\mathrm{PL}$, when the mobility is curvilinear. This is because, in the latter case, the ME spends in general more time in the communication area. This allows the PL protocol to use a lower $\delta$ when trying to discover the $\mathrm{ME}$, thus resulting in a lower energy saving by 2BD (as depicted in Table 4). However, due to the longer time spent by the ME in the discovery area, in the curvilinear scenario the $2 \mathrm{BD}$ protocol can use a lower low duty cycle, as compared to the linear scenario (resulting in a lower energy consumption). From Figure 4 and Figure 5 also conclude that, when the arrival time of the ME can be predicted with a high accuracy (e.g., the waiting time is one minute), in general the 2BD protocol is less energy efficient than PL, especially in the curvilinear scenario. Indeed, when the waiting time is very short, the major contribute to the energy consumption of the sensor node is given by $\delta_{H}$ (or $\delta$ in PL). Since the sensor node in 2BD has to compensate for the ME discovery latency given by the LRB-Discovery phase, in most of cases $\delta_{H}$ results to be (much) higher than $\delta$. Therefore, a higher energy consumption by the sensor node is observed when the waiting time is very short. Note that if the $\mathrm{ME}$ arrival time is known by the sensor node, the discovery process becomes unnecessary.

Therefore, as soon as the uncertainty (and hence, the waiting time) increases, the time spent by the sensor node in the discovery state increases, implying 2BD tends to be more and more energy efficient than PL. This is because 2BD uses a (much) lower duty cycle for most of the time while in the discovery state, as shown in Table 4. In particular, when the ME mobility pattern is random and sensor node is forced to be always in the discovery state, the energy savings achieved by 2BD may be very high. Figure 4 and Figure 5 also exhibit that, for a fixed waiting time, the energy savings provided by $2 \mathrm{BD}$ are much higher for large throughput values. This is because a larger throughput requires an earlier discovery of the $\mathrm{ME}$ and/or a higher percentage of detected contacts and, ultimately, a higher duty cycle. To this end, 2BD can take advantage of its hierarchical mechanism. As shown in Table 4, when the requested throughput changes from 40 to 60 Kbytes/contact, the residual contact time for both protocol increases from about $65 \%$ to about $90 \%$ (linear mobility). However, the duty cycle of PL increases from $1.5 \%$ to $6.5 \%$, while the low duty cycle of $2 \mathrm{BD}$ only passes from $0.9 \%$ to $1.4 \%$.

Please note that, when $\delta_{H}=\delta_{L}=\delta$ (i.e., all duty cycle values are equal), the performance of $2 \mathrm{BD}$ is different from that of PL. This is because, in 2BD, each beacon type (LRB and SRB) is emitted once every $2 * T_{b i}$ to keep the total number of beacons emitted equal to PL (as explained in Section 3). Therefore, when the MS is close enough to the sensing area, each LRB will be received once every $2 * T_{b i}$ time units. Conversely, in PL, the sensor node will receive each beacon once every $T_{b i}$. Hence, when duty cycle values are equal, the performance of $2 \mathrm{BD}$ is necessarily different from that of PL.

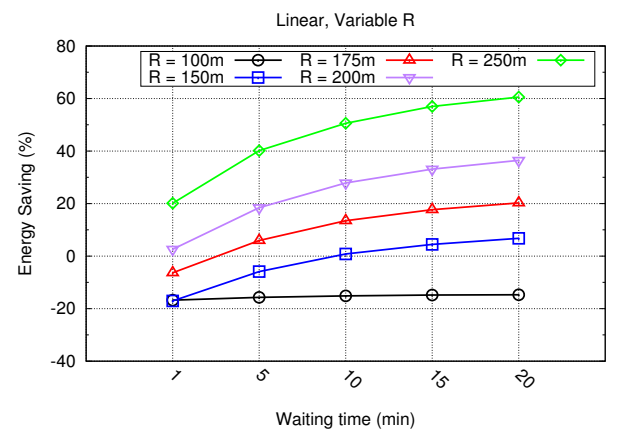

Figure 6: Energy savings of $2 \mathrm{BD}$ in function of $R$, Linear Scenario.

For the sake of brevity, in the following the results for the curvilinear scenario will be shown in tabular format only. Figure 6 depicts the impact of the discovery range $R$ on the energy efficiency of 2BD in the linear mobility scenario (note that PL is not affected by 
Table 5: Optimal duty cycle values in function of $R$, for linear (topside) and curvilinear (bottomside) $\mathrm{ME}$ mobility.

\begin{tabular}{|c|c|c|c|c|c|c|l|}
\hline $\mathrm{R}(\mathrm{m})$ & $\delta^{o p t}$ & $\delta_{L}^{o p t}$ & $\delta_{H}^{o p t}$ & $L$ & $H$ & $C R_{2 b d}$ & $C R_{p l}$ \\
\hline 100 & 1.5 & 1.7 & 5.8 & 5.89 & 6.88 & $64.37 \%$ & $66.48 \%$ \\
150 & 1.5 & 1.2 & 4.2 & 8.35 & 11.65 & $64.59 \%$ & $66.48 \%$ \\
175 & 1.5 & 1.0 & 5.6 & 10.26 & 13.23 & $64.85 \%$ & $66.48 \%$ \\
200 & 1.5 & 0.8 & 6.4 & 12.54 & 15.78 & $65.50 \%$ & $66.48 \%$ \\
250 & 1.5 & 0.6 & 7.6 & 14.82 & 18.04 & $64.59 \%$ & $66.48 \%$ \\
\hline $\mathrm{R}(\mathrm{m})$ & $\delta^{o p t}$ & $\delta_{L}^{o p t}$ & $\delta_{H}^{o p t}$ & $L$ & $H$ & $C R_{2 b d}$ & $C R_{p l}$ \\
\hline 100 & 0.8 & 1.2 & 4.0 & 8.35 & 10.32 & $55.43 \%$ & $59.21 \%$ \\
150 & 0.8 & 0.8 & 4.6 & 12.54 & 15.90 & $55.26 \%$ & $59.21 \%$ \\
175 & 0.8 & 0.7 & 5.1 & 12.54 & 15.90 & $55.26 \%$ & $59.21 \%$ \\
200 & 0.8 & 0.6 & 5.5 & 16.72 & 22.15 & $59.75 \%$ & $59.21 \%$ \\
250 & 0.8 & 0.5 & 6.2 & 20.12 & 28.02 & $59.43 \%$ & $59.21 \%$ \\
\hline
\end{tabular}

this parameter). From Figure 6 and Table 5 it emerges that, when the discovery area is shorter, there is no advantage of using $2 \mathrm{BD}$ with respect to $\mathrm{PL}$, since it cannot effectively leverage its hierarchical mechanism. This point is better explained by Table 5 . In fact, when $R=100$, the duty cycle used by $2 \mathrm{BD}$ and PL is $1.7 \%$ and $1.5 \%$, respectively. In line with expectations, as the $R$ value increases, 2BD shows better energy savings than PL, in both the linear and curvilinear scenarios. This follows from the fact that a further increase in the $R$ value allows to provide the same throughput by spending less energy (as the low duty cycle becomes lower), thus resulting in higher energy savings.

\subsubsection{Impact of ME speed and beacon period}

For the sake of simplicity, in the following we will assume that the ME speed $v^{\omega}(t)$ is constant throughout $w$. This is done without any loss of generality, as the variable speed parameter impacts only on the time spent by the $\mathrm{ME}$ in the communication and discovery area. Therefore, in order to evaluate the impact of speed (and consequently, the maximum nominal communication time $\tau_{c}^{\omega}$ ), Figure 7 shows the energy saving provided by $2 \mathrm{BD}$, with respect to $\mathrm{PL}$, as a function of the ME speed, for different values of the waiting time. The throughput bound for both protocols was set to $40 \mathrm{Kbytes}$ for $v=\{40,60\} \frac{\mathrm{km}}{\mathrm{h}}$, and to $35 \mathrm{Kbytes}$ for $v=70 \frac{\mathrm{km}}{\mathrm{h}}(40,30,28 \mathrm{Kbytes}$ in the curvilinear scenario). This is because, as the ME speed increases, $\tau_{c}^{\omega}$ becomes very short and a throughput of $40 \mathrm{Kbytes}$ per contact cannot be achieved. Similarly to Figure 4, we observe from Figure 7 that 2BD attains better performance with respect to PL as the ME speed increases and hence, the maximum nominal communication time $\tau_{c}^{\omega}$ decreases. In fact, Table 6 shows that, as the ME speed increases, the difference between the low duty cycle used by 2BD and the fixed duty cycle of PL becomes more and more significant - this is due to the shorter $\tau_{c}^{\omega}$. Therefore, as the ME speed increases, 2BD can use a (very much) lower low duty cycle than PL, in both the linear and the curvilinear scenarios. For example, when $v=70 \frac{\mathrm{km}}{\mathrm{h}}$, the low duty cycle used by 2BD is just $1.1 \%$, in contrast with PL that uses a duty cycle of $13.33 \%$ to obtain the same throughput performance. However, as stated beforehand, in the curvilinear scenario 2BD provides less energy saving with respect to the linear scenario, due to the fact that in this scenario the nominal contact time is larger, and thus, the PL protocol is advantaged and can use a lower duty cycle to obtain the same performance as the 2BD protocol.

Table 6: Optimal duty cycle values in function of $v(\mathrm{~km} / \mathrm{h})$, for linear (topside) and curvilinear (bottomside) ME mobility.

\begin{tabular}{|c|c|c|c|c|c|c|c|}
\hline$v$ & $\delta^{o p t}$ & $\delta_{L}^{o p t}$ & $\delta_{H}^{o p t}$ & $L$ & $H$ & $C R_{2 b d}$ & $C R_{p l}$ \\
\hline 40 & 1.5 & 0.8 & 6.4 & 12.54 & 15.78 & $65.50 \%$ & $66.48 \%$ \\
60 & 9.3 & 2.1 & 17.0 & 4.77 & 9.08 & $89.90 \%$ & $90.26 \%$ \\
70 & 13.33 & 1.1 & 20.4 & 4.58 & 7.60 & $92.95 \%$ & $92.11 \%$ \\
\hline$v$ & $\delta^{o p t}$ & $\delta_{L}^{o p t}$ & $\delta_{H}^{o p t}$ & $L$ & $H$ & $C R_{2 b d}$ & $C R_{p l}$ \\
\hline 40 & 0.8 & 0.6 & 5.5 & 16.72 & 22.15 & $59.75 \%$ & $59.21 \%$ \\
60 & 2.2 & 1.1 & 6.0 & 9.12 & 14.41 & $65.29 \%$ & $63.69 \%$ \\
70 & 3.8 & 1.5 & 6.7 & 6.69 & 12.66 & $69.38 \%$ & $74.95 \%$ \\
\hline
\end{tabular}

Table 7: Optimal duty cycle values in function of $T_{b i}(\mathrm{~ms})$, for linear (topside) and curvilinear (bottomside) ME mobility.

\begin{tabular}{|c|c|c|c|c|c|c|c|}
\hline$T_{B I}$ & $\delta^{o p t}$ & $\delta_{L}^{o p t}$ & $\delta_{H}^{o p t}$ & $L$ & $H$ & $C R_{2 b d}$ & $C R_{p l}$ \\
\hline 50 & 0.7 & 0.4 & 3.7 & 12.59 & 15.67 & $66.83 \%$ & $64.32 \%$ \\
100 & 1.5 & 0.8 & 6.4 & 12.54 & 15.78 & $65.50 \%$ & $66.48 \%$ \\
200 & 2.9 & 1.6 & 13.3 & 12.51 & 15.74 & $68.50 \%$ & $64.88 \%$ \\
\hline$T_{B I}$ & $\delta^{o p t}$ & $\delta_{L}^{o p t}$ & $\delta_{H}^{o p t}$ & $L$ & $H$ & $C R_{2 b d}$ & $C R_{p l}$ \\
\hline 50 & 0.4 & 0.3 & 2.6 & 16.81 & 22.26 & $60.58 \%$ & $59.70 \%$ \\
100 & 0.8 & 0.6 & 5.5 & 16.72 & 22.15 & $59.75 \%$ & $59.21 \%$ \\
200 & 2.3 & 1.3 & 7.4 & 15.41 & 22.40 & $56.22 \%$ & $59.81 \%$ \\
\hline
\end{tabular}

Finally, Figure 8 shows the impact of the beacon period on both protocols, supposing a throughput bound of 40 Kbytes per contact. As it can be observed, 2BD shows a different behavior for short and long waiting times. This is because, using a larger beacon period (e.g., passing from $50 \mathrm{~ms}$ to $200 \mathrm{~ms}$ ) makes the discovery process more difficult. Since the sensor node needs to increase its (low) duty cycle to guarantee the same performance, when the waiting time becomes longer, $2 \mathrm{BD}$ is more energy-efficient than PL, due to its hierarchical mechanism. Instead, when the waiting time is short, the additional energy consumed by $2 \mathrm{BD}$ in high duty cycle becomes predominant with respect to that consumed in low duty cycle and, overall, $\mathrm{PL}$ is more efficient than 2BD. However, unless the waiting time is very small, $2 \mathrm{BD}$ is always more efficient than PL, irrespective of the considered beacon period.

Furthermore, Figure 8 demonstrates that, when passing from a beacon period of $100 \mathrm{~ms}$ to $200 \mathrm{~ms}$, the energy saving provided by 2BD is nearly the same. In fact, since the bound on the system throughput is the same, irrespective of the beacon period, the LRB and SRB Discovery time (i.e., $L$ and $H$ ) needed by 2BD to guarantee the desired throughput is nearly constant (as emphasized in Table 7). Thus, since both protocols need to increase their duty cycles as the beacon period becomes larger, the energy saving provided by 2BD becomes independent of the beacon period used. 


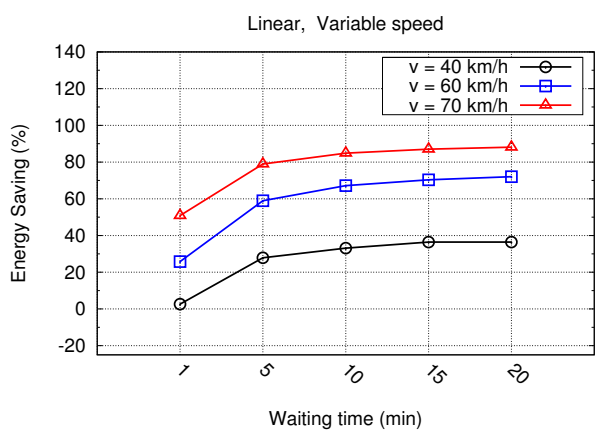

Figure 7: Energy saving of $2 \mathrm{BD}$ in function of $v$, Linear Scenario.

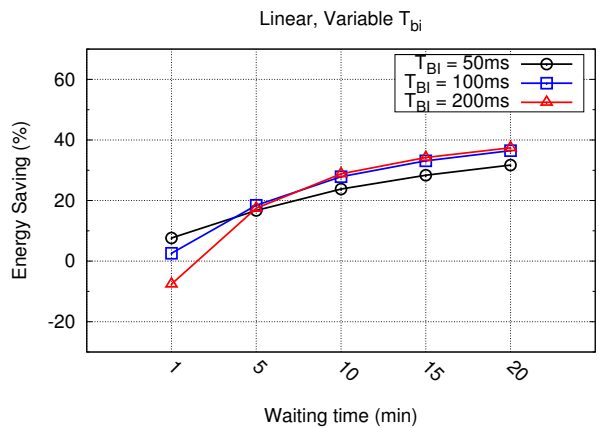

Figure 8: Energy saving of $2 \mathrm{BD}$ in function of $T_{b i}$, Linear Scenario.

\section{Conclusions}

In this paper, we have investigated and analyzed a hierarchical discovery protocol for WSN-MEs, namely Dual Beacon Discovery (2BD) protocol, and compared its performances with a classical ME discovery protocol (namely, PL) based on a single beacon. Our experimental results emphasize that $2 \mathrm{BD}$ can provide a significant energy saving with respect to a classic solution, especially when the discovery phase is relatively long and the application requires significant QoS bounds on the systems throughput. Thus, 2BD is perfectly suitable in situations in which the ME arrival time is unpredictable. However, it may be appealing also for scenarios where the ME mobility pattern is predictable, especially when the arrival time of the ME can be predicted with limited accuracy.

Due to lack of space, we have limited our analysis and discussions to a sparse network scenario. However, in a dense scenario, 2BD may cause false activations of sensor nodes, in sense that those located outside the communication range of the may receive a LRB, however, they will never receive a subsequent SRB. Obviously, false activations result in wastage of energy, and their number depends on the discovery range $R$ that is used. Our experimental results (not shown here for the sake of space) demonstrate that, with an appropriate setting of $\mathrm{R}$, the 2BD protocol outperforms PL in dense scenario as well. Furthermore, the number of false activations can be drastically reduced by exploiting information about the past history and/or the ME movement direction, if available. This will be part of our future research.

\section{ACKNOWLEDGMENT}

This research is partially supported by NSF grants CNS-0721951, CNS-1049652, and IIP-1242521. We are grateful to the anonymous reviewers for their insightful comments and constructive suggestions which helped us improve the quality of the paper significantly.

\section{REFERENCES}

[1] Wu, X., Chen, G. and Das, S.K., "Avoiding Energy Holes in Wireless Sensor Networks with Nonuniform Node Distribution," IEEE Transactions on Parallel and Distributed Systems, Vol. 19, No. 5, 2008.

[2] Akyildiz, I.F. and Stuntebeck, E., "Wireless Underground Sensor Networks: Research Challenges", Ad Hoc Networks, Vol. 4, No. 6, 2006.

[3] Di Francesco, M., Das, S.K., Anastasi, G., "Data Collection in Wireless Sensor Networks with Mobile Elements: A Survey", ACM Transactions on Sensor Networks, Vol.8, No. 1, 2011.

[4] Jain, S., Shah, R., Brunette, W., Borriello, G. and Roy, S., "Exploiting Mobility for Energy Efficient Data Collection in Wireless Sensor Networks", Mobile Networks and Applications, Vol. 11, No. 3, 2006.

[5] Shah, K., Di Francesco, M. Anastasi, G. and Kumar, M., "A Framework for Resource-Aware Data Accumulation in Sparse Wireless Sensor Networks", Computer Communications, Vol. 34, No. 17, 2011.

[6] Pering, T., Raghunathan, V., Want, R., "Exploiting Radio Hierarchies for Power-Efficient Wireless Device Discovery and Connection Setup", International Conference on VLSI Design, 2005.

[7] Jun, H., Ammar, M., Corner, M. and Zegura, E., "Hierarchical Power Management in Disruption Tolerant Networks Using Traffic-aware Optimization", Computer Communications, Vol. 32, No. 16, 2009.

[8] Kondepu, K., Anastasi, G., Conti, M., "Dual-Beacon MobileNode Discovery in Sparse Wireless Sensor Networks", IEEE ISCC, Corfu, Greece, 2011.

[9] Wu, X., Brown, K.N., and Sreenan, C.J., "SNIP: A Sensor NodeInitiated Probing Mechanism for Opportunistic Data Collection in Sparse Wireless Sensor Networks" First International Workshop on Cyber-Physical Networking Systems (CPNS'2011), Shangai, China, 2011.

[10] Anastasi, G., Conti, M. and Di Francesco, M., "Reliable and Energy-efficient Data Collection in Sparse Sensor Networks with Mobile Elements", Performance Evaluation, Vol. 66, No. 12, 2009.

[11] Galluccio, L., Morabito, G., and Palazzo, S., “Analytical Evaluation of a Tradeoff Between Energy Efficiency and Responsiveness of Neighbor Discovery in Self-organizing Ad Hoc Networks", IEEE Journal on Selected Areas in Communications, Vol. 22, No. 7, 2004.

[12] Wang, W., Motani, M., Srinivasan, V., “Opportunistic Energyefficient Contact Probing in Delay-Tolerant Applications", IEEE/ACM Transactions on Networking, Vol. 17, No. 5, 2009.

[13] Izumikawa, H., Pitkanen, M., Ott, J., Timm-Giel, A., and Bormann, C., "Energy-efficient Adaptive Interface Activation for Delay/Disruption Tolerant Networks", International Conference on Advanced Communication Technology (ICACT), 2010.

[14] Drula, C., Amza, C., Rousseau, F., and Duda, A., "Adaptive energy conserving algorithms for neighbor discovery in opportunistic Bluetooth networks", IEEE Journal on Selected Areas in Communications, Vol.25, No.1, 2007.

[15] Restuccia, F., Anastasi, G., Conti, M. and Das, S.K., "Performance Analysis of a Hierarchical Discovery Protocol for WSNs with Mobile Elements", IEEE WoWMoM, San Francisco, CA, USA, 2012

[16] Petrova, M., Riihijarvi, J., Mahonen, P. and Labella, S., "Performance Study of IEEE 802.15.4 Using Measurement and Simulations", IEEE WCNC, Las Vegas, NV, USA, 2006. 
[17] Basagni, S., Carosi, A., Melachrinoudis, E., Petrioli, C. and Wang, M., "Controlled Sink Mobility for Prolonging Wireless Sensor Networks Lifetime", ACM Wireless Networks, Vol. 14, No. 6, 2008

[18] Anastasi, G., Conti, M., Monaldi, E., and Passarella, A., "An Adaptive Data-transfer Protocol for Sensor Networks with Data Mules", IEEE WoWMoM, Helsinki, Finland, 2007.

[19] Avriel, M., "Nonlinear Programming: Analysis and Methods". Dover Publishing. IPLN 0-486-43227-0, 2003.

[20] IEEE 802.15.4 standard. http://standards.ieee.org/getieee802/ download/802.15.4-2006.pdf.

[21] Chipcon, 2.4 GHz IEEE 802.15.4/ZigBee-Ready RF Transceiver, Chipcon Products from Texas Instruments, 2004, CC2420 Data Sheet.

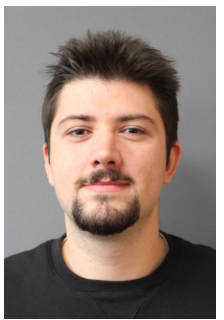

Francesco Restuccia is a first-year graduate student at the Department of Computer Science and Engineering of the University of Texas, Arlington, TX, USA. Currently, he is pursuing his Ph.D. in Computer Science under the supervision of Prof. Dr. Sajal K. Das, as a member of the Center for Research in Wireless Mobility and Networking (CReWMaN). He received his B.S. and M.S. in Computer Engineering from the University of Pisa, Italy, both summa cum laude, in 2009 and 2011, respectively. Before joining UTA, he has been a Research Assistant at IIT-CNR, Pisa, Italy. His research interests include analysis and modeling of complex systems, pervasive computing, wireless and sensor networks.

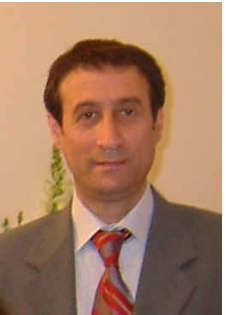

Giuseppe Anastasi is a Full Professor and the Deputy Director of the Department of Information Engineering at the University of Pisa, Italy. His research interests include pervasive computing systems, sensor networks, sustainable computing, and computing for sustainability. $\mathrm{He}$ is the founding co-chair of the Pervasive Computing Networking Laboratory (PerLab), and has contributed to many research programs funded by both national and international institutions. $\mathrm{He}$ is a $\mathrm{co}-$ editor of two books: Advanced Lectures in Networking (LNCS 2497, Springer, 2002) and Methodologies and Technologies for Networked Enterprises (LNCS 7200, Springer, 2012). He has published over 100 research papers in the area of computer networking and pervasive computing. Giuseppe is an Associate Editor of Sustainable Computing (SUSCOM) and an Area Editor of Pervasive and Mobile Computing (PMC). He has served as Area Editor of Computer Communications (ComCom, 2008-10), General Co-chair of IEEE WoWMoM 2005, Program Chair of IFIP/IEEE SustainIT 2012, IEEE PerCom 2010 and IEEE WoWMoM 2008, Vice Program Chair of IEEE MASS 2007, Workshops Chair of IEEE PerCom 2006, IEEE WoWMoM 2006, and IEEE ICCCN 2007. He is one of the cofounders of the IFIP/IEEE International Conference on Sustainable Internet and ICT for Sustainability (SustainIT) and of the IEEE International Workshop on Sensor Networks and Systems for Pervasive Computing (PerSeNS), co-located with IEEE PerCom. He has been a member of the IEEE Computer Society since 1994.

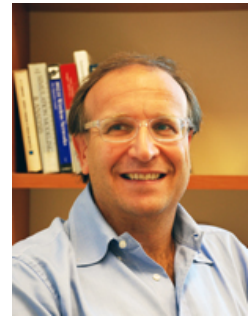

Marco Conti is a research director of the Italian National Research Council (CNR) and director of the CNR department of Engineering, ICT and Technologies for Energy and Transports. He published in journals and conference proceedings more than 300 papers related to design, modelling, and performance evaluation of computer-network architectures and protocols. He coauthored the book Metropolitan Area Networks (1997) and is co-editor of the books Mobile Ad Hoc Networking (2004), Mobile Ad Hoc Networks:From Theory to Reality (2007), and Mobile Ad Hoc Networking: Cutting Edge Directions (2012). He is the Editor-in-Chief of Elsevier's Computer Communications (COMCOM) Journal, and Associate Editor-in-Chief of Elsevier's Pervasive and Mobile Computing (PMC) Journal. He is the Chair of the IFIP Working Group 6.3 Performance of Communication Systems. He served as general/program chair for several conferences, including IEEE PerCom, IEEE WoWMoM, IEEE MASS, ACM MobiHoc, and IFIP TC6 Networking. He is the cofounder of the IFIP/IEEE SustainIT (Sustainable Internet and ICT for Sustainability) conference and (co)founder of ACM REALMAN (Multi-hop Ad Hoc Networks: from theory to reality), ACM MobiOpp (Mobile Opportunistic Networking) and IEEE AOC (Autonomic and Opportunistic Communications) workshop series.

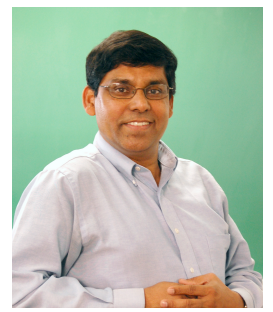

Sajal K. Das is a University Distinguished Scholar Professor of Computer Science and Engineering and the Founding Director of the Center for Research in Wireless Mobility and Networking (CReWMaN) at the University of Texas at Arlington. During 2008-2011, he served the US National Science Foundation (NSF) as a Program Director in the Division of Computer Networks and Systems. In 2012 he was selected as E.T.S. Walton Fellow by the Science Foundation of Ireland. His current research interests include wireless and sensor networks, mobile and pervasive computing, cloud computing, cyber-physical systems and smart environments, security and privacy, biological and social networks, applied graph theory and game theory. He has published over 500 papers and 47 invited book chapters in these areas. He holds five US patents in wireless networks and mobile Internet, and coauthored three books: Smart Environments: Technology, Protocols, and Applications (Wiley, 2005), "Mobile Agents in Distributed Computing and Networking" (Wiley, 2012), and "Handbook on Securing Cyber-Physical Critical Infrastructure: Foundations and Challenges" (Morgan Kaufmann, 2012). Dr. Das is a recipient of the IEEE Computer Society Technical Achievement Award (2009) for pioneering contributions to sensor networks, and several Best Paper Awards including those at IEEE SmartGridComm12, QShine09, EWSN08, IEEE PerCom06, and ACM MobiCom99. he serves as the Founding Editor-in-Chief of Elsevier's Pervasive and Mobile Computing (PMC) journal, and also as an associate editor of several other journals. He is a founder of the IEEE WoWMoM, IEEE PerCom and ICDCN conferences. He has served as General and Technical Program Chair as well as TPC member of numerous IEEE and ACM conferences. 


\section{APPENDIX}

\section{A. Derivation of $\tau_{d-c^{\prime}}^{\omega} \tau_{d}^{\omega}$, and $\tau_{c}^{\omega}$}

Let us suppose that all curves $\omega$ are defined in a twodimensional Cartesian Coordinate System (C.C.S.). Let $\mathcal{V} \triangleq\{\mathcal{X}, \mathcal{Y}\} \in \mathbb{R}^{2}$ be a C.C.S. centered in $\mathcal{O}=\{0,0\}$. Let $\mathcal{C}($ resp. $\mathcal{D}) \in \mathcal{V}$ be a curve describing the boundaries of the communication (resp. discovery) area. Let $C_{\omega}^{i n}$ and $C_{\omega}^{\text {out }}$ (resp. $D_{\omega}^{\text {in }}$ and $D_{\omega}^{\text {out }}$ ) be the points in $\mathcal{V}$ in which the ME enters and exits the communication (resp. discovery) area. In order to simplify the presentation, we suppose that the ME has only one ingress / egress point in the communication and discovery area. By definition, we can derive $C_{\omega}^{i n}$ and $C_{\omega}^{\text {out }}$ (resp. $D_{\omega}^{\text {in }}$ and $D_{\omega}^{\text {out }}$ ) as the nearest points from origin present in the intersection of $\mathcal{C}(\operatorname{resp} . \mathcal{D})$ and $\omega$. That is

$$
\begin{gathered}
\Lambda_{C} \triangleq\{|\lambda|, \lambda \in \mathcal{C} \cap \omega\} \\
\Lambda_{D} \triangleq\{|\lambda|, \lambda \in \mathcal{D} \cap \omega\} \\
C_{\omega}^{i n}=\min \Lambda_{C} \\
C_{\omega}^{\text {out }}=\min \left(\Lambda_{C}-C^{i n}\right) \\
D_{\omega}^{\text {in }}=\min \Lambda_{D} \\
D_{\omega}^{\text {out }}=\min \left(\Lambda_{D}-D^{\text {in }}\right)
\end{gathered}
$$

The following Lemma holds.

Lemma 1. The distance covered by the ME from $D_{\omega}^{i n}$ to $C_{\omega}^{i n}$, from $C_{\omega}^{i n}$ to $C_{\omega}^{\text {out }}$ and from $D_{\omega}^{i n}$ to $D_{\omega}^{\text {out }}$ (respectively, $S_{D-C}^{\omega}, S_{C}^{\omega}$ and $\left.S_{D}^{\omega}\right)$ is

$$
\begin{gathered}
S_{D-C}^{\omega}=\int_{D_{\omega}^{\text {in }}}^{C_{D}^{i n}} \sqrt{1+\omega^{\prime}(x)^{2}} d x \\
S_{C}^{\omega}=\int_{C_{\omega}^{\text {in }}}^{C_{\text {out }}^{\text {in }}} \sqrt{1+\omega^{\prime}(x)^{2}} d x \\
S_{D}^{\omega}=\int_{D_{\omega}^{\text {Din }}}^{D_{\omega}^{\text {out }}} \sqrt{1+\omega^{\prime}(x)^{2}} d x
\end{gathered}
$$

Proof.

$$
\begin{gathered}
d s^{2}=d x^{2}+d y^{2} \quad \frac{d s^{2}}{d x^{2}}=1+\frac{d y^{2}}{d x^{2}} \\
d s=\sqrt{1+\left(\frac{d y}{d x}\right)^{2}} \quad s=\int_{a}^{b} \sqrt{1+\left[f^{\prime}(x)\right]^{2}} d x
\end{gathered}
$$

Equation (1) holds only if we suppose $\omega$ being a continuous and derivable function. A general formula for calculating the distances $S_{D-C}^{\omega}, S_{C}^{\omega}$ and $S_{D}^{\omega}$ considering a general curve $\omega$ is provided in Appendix B. Supposing $t=0$ as the time in which the ME enters into the discovery area, let us define $t_{C}^{\text {in, } \omega}, t_{C}^{\text {out }, \omega}$ and $t_{D}^{\text {out }, \omega}$ as the time instants in which the ME enters and exits the communication and discovery areas, respectively. Defining $\bar{v}_{D-C}^{\omega}, \bar{v}_{C}^{\omega}$ and $\bar{v}_{D}^{\omega}$ as the ME's average speed from $t=0$ to $t_{C}^{i n, \omega}, t_{C}^{o u t, \omega}$, and $t_{D}^{\text {out }, \omega}$, respectively, we can derive the desired quantities as

$$
t_{C}^{i n, \omega}=\frac{S_{D-C}}{\bar{v}_{D-C}^{\omega}}, t_{C}^{\text {out }, \omega}=\frac{S_{C}}{\bar{v}_{C}^{\omega}}, t_{D}^{\text {out }, \omega}=\frac{S_{D}}{\bar{v}_{D}^{\omega}}
$$

Thus, we can easily derive $\tau_{d-c}^{\omega}=t_{C}^{i n, \omega}, \tau_{d}^{\omega}=t_{D}^{o u t, \omega}$, and $\tau_{c}^{\omega}=t_{C}^{o u t, \omega}-t_{C}^{i n, \omega}$.

\section{B. Derivation of the distances for a generic curve $\omega$}

As far as a general curve $\omega \in \Omega$ is concerned, we can derive the quantities $S_{D-C}^{\omega}, S_{C}^{\omega}$ and $S_{D}^{\omega}$ (resp. the time spent by the $\mathrm{ME}$ in the discovery area before entering the communication area, in the communication area, and in the discovery area overall) as follows. As in Section 4.1, let us suppose that all curves $\omega$ are defined in a two-dimensional Cartesian Coordinate System (C.C.S.), and let $\mathcal{V} \triangleq\{\mathcal{X}, \mathcal{Y}\} \in \mathbb{R}^{2}$ be a C.C.S. centered in $\mathcal{O}=\{0,0\}$. Also, let us define as $\mathcal{V}_{\omega} \subseteq \mathcal{V}$ the subspace defined by all the points belonging to the curve $\omega$. We are interested to calculate the distance between two generic points $\theta \triangleq\left(x_{\theta}, y_{\theta}\right), \theta^{\prime} \triangleq\left(x_{\theta}^{\prime}, y_{\theta}^{\prime}\right) \in \mathcal{V}_{\omega}$ such as $x_{\theta}^{\prime}>x_{\theta}$. Let us define $\Delta_{x}$ as a space quantization parameter, and $N_{\Delta}=\left\lfloor\frac{x_{\theta}^{\prime}-x_{\theta}}{\Delta_{x}}\right\rfloor$. We define $\omega_{i}\{x\} \in \mathcal{V}_{\omega}$ as the point individuated by the following relation:

$$
\omega_{i}\{\theta\} \triangleq\left\{x_{\theta}+i \cdot \Delta_{x}, \omega\left(x_{\theta}+i \cdot \Delta_{x}\right)\right\}, i=0, \ldots, N_{\Delta}
$$

Finally, the distance $S\left\{\theta, \theta^{\prime}\right\}$ between the two points $\theta$ and $\theta^{\prime}$ can be approximated by the sum of the distances between the points $\omega_{i}\{\theta\}$ and $\omega_{i+1}\{\theta\}$, i.e.,

$$
S\left\{\theta, \theta^{\prime}\right\}=\sum_{i=0}^{N_{\Delta}-1} d\left(\omega_{i}\{\theta\}, \omega_{i+1}\{\theta\}\right)
$$

By using Equation (2), we can compute $S_{D-C}^{\omega}, S_{C}^{\omega}$ and $S_{D}^{\omega}$ as $S_{D-C}^{\omega}=S\left\{D_{\omega}^{i n}, C_{\omega}^{i n}\right\}, S_{C}^{\omega}=S\left\{C_{\omega}^{i n}, C_{\omega}^{o u t}\right\}, S_{D}^{\omega}=$ $S\left\{D_{\omega}^{\text {in }}, D_{\omega}^{\text {out }}\right\}$.

\section{B. DTMC State diagram for $t_{0}^{S}<t_{0}^{L}$}

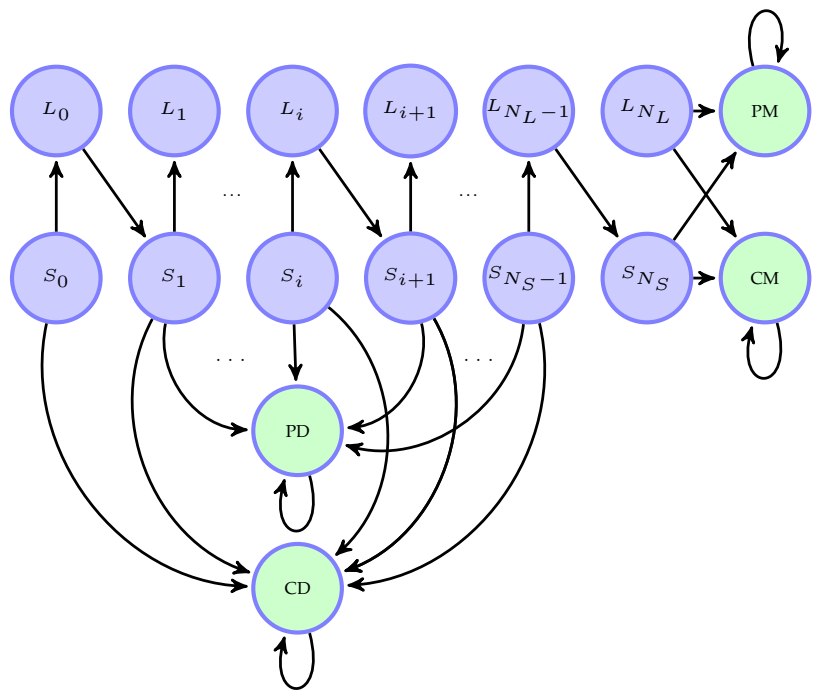

Figure 1: DTMC State diagram for $t_{0}^{S}<t_{0}^{L}$.

Figure 1 shows the state transition diagram when $t_{0}^{S}<$ $t_{0}^{L}$. In detail, the initial state is $S_{0}$. From a generic state $L_{i}$, the system always evolves to state $S_{i+1}$, regardless of the $i$-th LRB reception status. From a generic state $S_{i}$, the system evolves into state:

- $L_{i}$, if the $i$-th SRB has been missed because the radio is OFF, i.e., $R S\left(t_{i}^{S}\right)=O F F$, or a transmission error has occurred, which occurs with probability $p\left(t_{i}^{S}\right)$;

- CD, if an LRB has already been received, i.e., $R_{L R B}\left(t_{i}^{L}\right)=$ 'True';

- PD, otherwise. 


\section{Proof of Claim 1}

As LRBs are not affected by message loss, given a certain initial radio state $\left(R S_{0}, r_{0}\right)$ and $t_{0}^{S}$, the time of the first LRB reception by sensor node, i.e., $t_{R-L R B}$, can be a priori derived as the first time in which the ME transmits a LRB and the radio of the sensor node is in ON state. Therefore, we can define an auxiliary function $R_{L R B}(t)$, that assumes the value True if a LRB has already been received at time $t$, and False otherwise. Since the time of the first LRB reception is known, and because of the duty cycle, it follows that $R S\left(t_{k}\right)$ always evolves in a deterministic and periodic way. In detail, when sensor node is waiting for a LRB (i.e., $R_{L R B}\left(t_{k}\right)=$ False), the radio state is periodic with period equal to $T_{L D C}$. Thus, in this case, it is possible to derive $R S\left(t_{k}\right)$ by comparing the remainder of the ratio between $t_{k}$ and $T_{L D C}$ against the initial residual time $r_{0}$. Upon the reception of a LRB (at time $\left.t_{R-L R B}\right)$, sensor node switches immediately to high duty cycle and waits for a SRB. From this time on, $R S\left(t_{k}\right)$ continues to be periodic as well, however, the duty cycle period is equal to $T_{H D C}$. Therefore, in case of high duty cycle (i.e., $R_{L R B}\left(t_{k}\right)=$ True), it is possible to derive $R S\left(t_{k}\right)$ by comparing the remainder of the ratio between $t_{k}-t_{R-L R B}$ and $T_{L D C}$ against the active time $T_{O N}$. Note that $T_{O N}$ does not change from low to high to duty cycle, as the beacon emission period $T_{b i}$ is independent from the current duty cycle of the sensor node.

\section{Optimization algorithm of $2 B D$}

Initially, the algorithm chooses a random point $\delta^{(0)}$ : $\theta\left\{\delta^{(0)}\right\} \geq \theta_{\text {min }}$. Then, the algorithm explores the search space in a neighborhood $d \delta$ of $\delta^{(k)}$ by considering all possible points $\hat{\delta}: \theta\{\hat{\delta}\} \geq \theta_{\min }$ having Euclidean distance $\left\|\hat{\delta}^{(k)}-\delta^{(k)}\right\| \in\{d \delta, \sqrt{2} \cdot d \delta\}$. Subsequently, a new point $\delta^{(k+1)}$ is chosen following the steepest descent criterion, i.e., the algorithm chooses the point by which the energy minimization is maximized. Finally, the algorithm terminates when none of the explored points reduces the energy obtained at the previous step, i.e., the energy $\bar{E}_{b}\left(\delta^{(k-1)}\right)$ obtained in the previous step differs from all the energies calculated in the current step less than a pre-defined quantity $\epsilon$. As a consequence, the algorithm terminates returning the point obtained in the previous step $k-1$. Since, by definition of the algorithm, $\delta^{(0)}>\delta^{(1)}>\delta^{(2)}>\cdots$, the algorithm converges to a strong local minimum of the energy function. Given the search space is limited (i.e., $\left.\delta_{L}, \delta_{H} \in(0,1]\right)$, a global solution may (eventually) be found by using an exhaustive search over the search space. 